\title{
Highly Enantioselective Direct Conjugate Addition of Ketones to Nitroalkenes Promoted by A Chiral Primary Amine-Thiourea Catalyst
}

\author{
Hongbing Huang and Eric N. Jacobsen*
}

Supporting Information

General Information: Nuclear magnetic resonance (NMR) spectra were recorded on a Varian Inova-500 or a Varian Mercurry-400. Chemical shifts are reported in parts per million using the solvent resonance internal standard (chloroform, 7.26 and $77.0 \mathrm{ppm}$, unless specified otherwise). Data are reported as follows: chemical shift, multiplicity $($ app $=$ apparent, par obsc $=$ partially obscured, ovrlp = overlapping, $\mathrm{s}=$ singlet, $\mathrm{d}=$ doublet, $\mathrm{t}=$ triplet, $\mathrm{q}=$ quartet, $\mathrm{m}=$ multiplet, $\mathrm{br}=$ broad, $\mathrm{abq}=\mathrm{ab}$ quartet), coupling constant, and integration. FT-IR infrared spectra were recorded on a Mattson Galaxy Series FTIR 3000 spectrometer. Optical rotations were measured on a Jasco DIP 370 digital polarimeter using a $2 \mathrm{~mL}$, $1 \mathrm{dm}$ cell, and are reported as $[\alpha]_{\mathrm{D}}$ (concentration in grams/100 $\mathrm{mL}$ solvent). Melting points were measured on a Mel-Temp apparatus, and are uncorrected. The mass spectroscopic data were obtained at the Harvard University Mass Spectrometry Facility. Chiral HPLC analysis was performed on a HewlettPackard 1050 instrument. Chiral GC analysis was performed on a Hewlett-Packard 5890 gas chromatography. All reactions were carried out in oven-dried glassware under a nitrogen atmosphere unless otherwise noted. Analytical thin layer chromatography was performed on 0.25mm EMD Chemical silica gel plates. Silica gel 60 (230-400 mesh) from EMD Chemicals was used for flash chromatography.

\section{Experimental Procedures for Catalyzed Michael Addition of Ketone to Nitroolefin:}

General Procedure A. The nitroolefin $(1.0 \mathrm{mmol})$ and catalyst $1(0.1 \mathrm{mmol})$ were placed in a $1.5 \mathrm{~mL}$ vial equipped with a Teflon-coated stirbar. Toluene $(50 \mu \mathrm{L})$ was added under $\mathrm{N}_{2}$, followed by the addition of ketone $(1.1 \mathrm{mmol})$. The vial was capped and the resulting mixture was stirred for $24 \mathrm{~h}$. The reaction progress was monitored by ${ }^{1} \mathrm{H}$ NMR analysis. Upon complete consumption of the nitroolefin, the crude reaction mixture was applied to silica gel and product isolated by flash chromatography.

General Procedure B. The nitroolefin $(1.0 \mathrm{mmol})$, benzoic acid $(0.1 \mathrm{mmol})$ and catalyst $1(0.1$ $\mathrm{mmol})$ were placed in a $1.5 \mathrm{~mL}$ vial equipped with a Teflon-coated stirbar. Toluene $(50 \mu \mathrm{L})$ was added under $\mathrm{N}_{2}$, followed by the addition of ketone $(5.0 \mathrm{mmol})$. The vial was capped and the resulting mixture was stirred for $24 \mathrm{~h}$. The reaction progress was monitored by ${ }^{1} \mathrm{H}$ NMR analysis. Upon complete consumption of the nitroolefin, the crude reaction mixture was applied to silica gel and product isolated by flash chromatography.

General Procedure C. The nitroolefin $(0.5 \mathrm{mmol})$ and catalyst $1(0.05 \mathrm{mmol})$ were placed in a $1.5 \mathrm{~mL}$ vial equipped with a Teflon-coated stirbar. Toluene $(250 \mu \mathrm{L})$ was added under $\mathrm{N}_{2}$, followed by the addition of ketone $(2.0 \mathrm{mmol})$. The resulting mixture was stirred at $\mathrm{rt}$, and an addition portion of catalyst $(0.05 \mathrm{mmol}$ in $80 \mu \mathrm{L}$ of toluene) was added after $12 \mathrm{~h}$. The resulting mixture was stirred for additional $24 \mathrm{~h}$. The reaction progress was monitored by ${ }^{1} \mathrm{H}$ NMR 
analysis. Upon complete consumption of the nitroolefin, the crude reaction mixture was applied to silica gel and product isolated by flash chromatography.

General Procedure D. The catalyst $1(0.1 \mathrm{mmol})$ was placed in a $1.5 \mathrm{~mL}$ vial equipped with a Teflon-coated stirbar. Toluene $(50 \mu \mathrm{L})$ was added under $\mathrm{N}_{2}$, followed by the addition of ketone $(2.5 \mathrm{mmol})$. A solution of nitroolefin $(0.5 \mathrm{mmol})$ in toluene $(200 \mu \mathrm{L})$ was added via syringe pump over $20 \mathrm{~h}$. After the completion of the addition of nitroolefin, the resulting mixture was stirred for additional $24 \mathrm{~h}$. The reaction progress was monitored by ${ }^{1} \mathrm{H}$ NMR analysis. Upon complete consumption of the nitroolefin, the crude reaction mixture was applied to silica gel and product isolated by flash chromatography.

\section{Catalyst screen.}
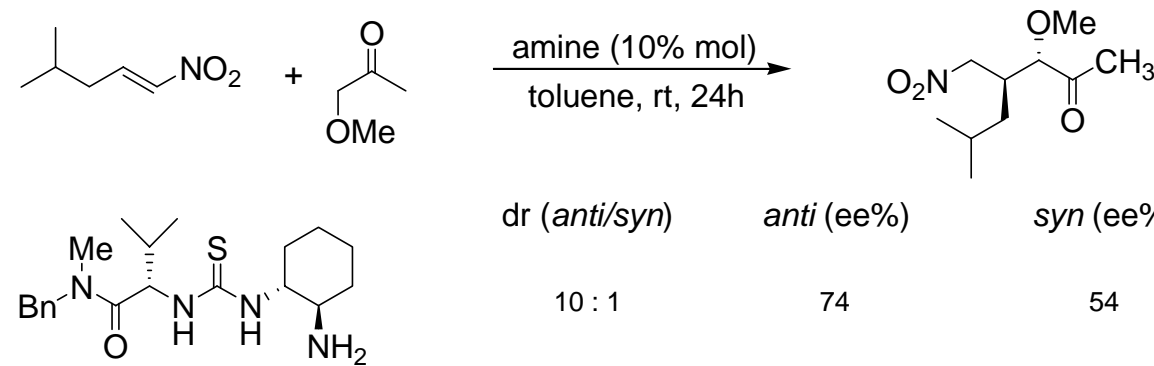

$\mathrm{dr}(\mathrm{anti} / \mathrm{syn}) \quad$ anti $(\mathrm{ee} \%) \quad$ syn $(\mathrm{ee} \%)$

$10: 1 \quad 74 \quad 54$<smiles>CN(Cc1ccccc1)C(=O)C(NC(=S)N[C@H]1CCCC[C@H]1N)c1ccccc1</smiles>

$8: 1$

82

76<smiles>CC(NC(=S)[NH2+]C1CCCCC1N)C(=O)N(C)Cc1ccccc1</smiles>

$6: 1$

71

40<smiles>N[C@H]1CCCCC1[NH2+]C(=S)Nc1cc(C(F)(F)F)cc(C(F)(F)F)c1</smiles>

$4: 1 \quad 56 \quad 40$

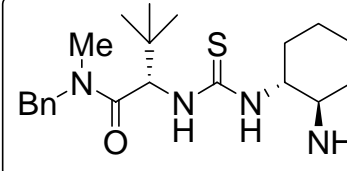

$10: 1$

85

72<smiles>CN(Cc1ccccc1)C(=O)[C@H](NC(=S)N[C@H]1CCCC[C@H]1N)C(C)(C)C</smiles>

$3.5: 1$

$-54$

$-89.5$

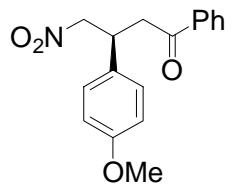

Prepared from trans-4-methoxy- $\beta$-nitrostyrene $(180 \mathrm{mg}, 1.0 \mathrm{mmol})$ and acetophenone $(1.1 \mathrm{mmol}, 128 \mu \mathrm{L})$ according to General Procedure A, affording $212 \mathrm{mg}$ of white solid after chromatography $\left(4: 1\right.$, hexanes:EtOAc). $[\alpha]_{\mathrm{D}}{ }^{20}-23.4^{\mathrm{o}}$ (c 1.42, $\mathrm{CHCl}_{3}$ ); mp 70.5-72.5 ${ }^{\circ} \mathrm{C}$; IR (KBr) 3033, 2936, 2839, 1681, 1549, 1255, 1180, 1029, 760, 691, $560 \mathrm{~cm}^{-1} ;{ }^{1} \mathrm{H}$ NMR (400 MHz, $\left.\mathrm{CDCl}_{3}\right) \delta 7.92(\mathrm{~d}, \mathrm{~J}=5.9 \mathrm{~Hz}, 2 \mathrm{H}), 7.58(\mathrm{t}$, $\mathrm{J}=7.3,1 \mathrm{H}), 7.46(\mathrm{t}, \mathrm{J}=8.1 \mathrm{~Hz}, 2 \mathrm{H}), 7.22(\mathrm{~d}, \mathrm{~J}=8.8 \mathrm{~Hz}, 2 \mathrm{H}), 6.87(\mathrm{~d}, \mathrm{~J}=8.8 \mathrm{~Hz}, 1 \mathrm{H}), 4.83(\mathrm{dd}$, $\mathrm{J}=6.95,12.3 \mathrm{~Hz}, 1 \mathrm{H}), 4.67(\mathrm{dd}, \mathrm{J}=8.1,12.4 \mathrm{~Hz}, 1 \mathrm{H}), 4.18(\mathrm{~m}, 1 \mathrm{H}), 3.78(\mathrm{~s}, 3 \mathrm{H}), 3.48(\mathrm{abq}, \mathrm{J}=$ 
6.6, $16.5 \mathrm{~Hz}, 2 \mathrm{H}) ;{ }^{13} \mathrm{C} \mathrm{NMR}\left(75 \mathrm{MHz}, \mathrm{CDCl}_{3}\right) \delta 197.0,159.0,136.4,133.5,131.0,128.7,128.5$, 128.0, 114.4, 79.8, 55.2, 41.6, 38.6; LRMS (CI) m/z calcd for $\mathrm{C}_{17} \mathrm{H}_{17} \mathrm{NO}_{4} 299$, found 299 .

$\mathrm{O}_{2} \mathrm{~N} \quad \mathrm{Ph}$ Prepared from trans- $\beta$-nitrostyrene $(150 \mathrm{mg}, 1.0 \mathrm{mmol})$ and acetophenone $(1.1$ mmol, $128 \mu \mathrm{L}$ ) according to General Procedure A, affording $223 \mathrm{mg}$ (83\% yield) of white solid after chromatography $\left(4: 1\right.$, hexanes:EtOAc). $[\alpha]_{\mathrm{D}}{ }^{20}-23.2^{\circ}$ (c 2.51, $\mathrm{CHCl}_{3}$ ); mp 91-92.5 ${ }^{\circ} \mathrm{C}$; IR (KBr) 3081, 3060, 3029, 2921, 1688, 1546, 1373, 1269, 1225, 1184, 703, 686, $560 \mathrm{~cm}^{-1} ;{ }^{1} \mathrm{H}$ NMR $\left(400 \mathrm{MHz}, \mathrm{CDCl}_{3}\right) \delta 7.93(\mathrm{~m}, 2 \mathrm{H}), 7.60(\mathrm{~m}, 1 \mathrm{H}), 7.48(\mathrm{~m}, 2 \mathrm{H})$, 7.36-7.25 (m, 5H), $6.87(\mathrm{~d}, \mathrm{~J}=8.8 \mathrm{~Hz}, 1 \mathrm{H}), 4.86(\mathrm{dd}, \mathrm{J}=6.95,12.4 \mathrm{~Hz}, 1 \mathrm{H}), 4.72$ (dd, J = 8.1, $12.4 \mathrm{~Hz}, 1 \mathrm{H}), 4.27(\mathrm{~m}, 1 \mathrm{H}), 3.52(\mathrm{~m}, 2 \mathrm{H}), 3.48(\mathrm{abq}, \mathrm{J}=6.6,16.5 \mathrm{~Hz}, 2 \mathrm{H}) ;{ }^{13} \mathrm{C}$ NMR $(75 \mathrm{MHz}$, $\left.\mathrm{CDCl}_{3}\right) \delta 196.8,139.1,136.3,133.6,129.0,128.7,128.0,127.9,127.4,79.5,41.5,39.2$; LRMS (CI) $\mathrm{m} / \mathrm{z}$ calcd for $\left[\mathrm{M}+\mathrm{NH}_{4}\right] 287$, found 287 .

$\mathrm{O}_{2} \mathrm{~N} \mathrm{CH}_{3}$ Prepared from trans- $\beta$-nitrostyrene $(150 \mathrm{mg}, 1.0 \mathrm{mmol})$ and acetone $(5.0 \mathrm{mmol}$, $368 \mu \mathrm{L}$ ) according to General Procedure B, affording 193mg (93\% yield) of white solid after chromatography $\left(4: 1\right.$, hexanes:EtOAc). $[\alpha]_{\mathrm{D}}{ }^{20}+2.4^{\circ}$ (c 1.03 , $\mathrm{CHCl}_{3}$ ); mp 120-122.5 ${ }^{\circ} \mathrm{C}$; ( IR (KBr) 3064, 3035, 2968, 2948, 2919, 2902, 1714, 1548, 1384, 1361, 1163, 758, 696, $548 \mathrm{~cm}^{-1}$; ${ }^{1} \mathrm{H}$ NMR $\left(400 \mathrm{MHz}, \mathrm{CDCl}_{3}\right) \delta 7.35(\mathrm{~m}, 2 \mathrm{H}), 7.32(\mathrm{~m}, 1 \mathrm{H}), 7.22$ $(\mathrm{m}, 2 \mathrm{H}), 4.71(\mathrm{dd}, \mathrm{J}=7.3,12.5 \mathrm{~Hz}, 1 \mathrm{H}), 4.62(\mathrm{dd}, \mathrm{J}=7.8,12.2 \mathrm{~Hz}, 1 \mathrm{H}), 4.04(\mathrm{~m}, 1 \mathrm{H}), 2.93(\mathrm{~d}, \mathrm{~J}$ $=7.32,1 \mathrm{H}), 2.12(\mathrm{~s}, 3 \mathrm{H}) ;{ }^{13} \mathrm{C} \mathrm{NMR}\left(75 \mathrm{MHz}, \mathrm{CDCl}_{3}\right) \delta 205.4,138.8,129.0,127.9,127.3,79.4$, $55.5,46.1,39.0,30.4$; LRMS (CI) $\mathrm{m} / \mathrm{z}$ calcd for $\left[\mathrm{M}+\mathrm{NH}_{4}\right] 225$, found 225 .

$\mathrm{O}_{2} \mathrm{~N} \quad \mathrm{CH}_{3} \quad$ Prepared from trans-4-methoxy- $\beta$-nitrostyrene $(180 \mathrm{mg}, 1.0 \mathrm{mmol})$ and acetone $(5.0 \mathrm{mmol}, 368 \mu \mathrm{L})$ according to General Procedure B, affording $208 \mathrm{mg}$ of white solid after chromatography $\left(19: 10\right.$, hexanes:EtOAc). $[\alpha]_{\mathrm{D}}{ }^{20}-1.5^{\circ}$ (c 1.32, oмe $\left.\quad \mathrm{CHCl}_{3}\right) ; \mathrm{mp} 93.5-94.5{ }^{\circ} \mathrm{C}$; IR ( $\left.\mathrm{KBr}\right) 3028,3002,2960,2901,1715,1550,1517$, 1260, 1180, 1033, 813, $554 \mathrm{~cm}^{-1} ;{ }^{1} \mathrm{H}$ NMR $\left(400 \mathrm{MHz}, \mathrm{CDCl}_{3}\right) \delta 7.14(\mathrm{~d}, \mathrm{~J}=8.4 \mathrm{~Hz}, 2 \mathrm{H}), 6.86$ $(\mathrm{d}, \mathrm{J}=8.8 \mathrm{~Hz}, 2 \mathrm{H}), 4.68(\mathrm{dd}, \mathrm{J}=6.6,12.3 \mathrm{~Hz}, 1 \mathrm{H}), 4.58(\mathrm{dd}, \mathrm{J}=7.7,12.1 \mathrm{~Hz}, 1 \mathrm{H}), 3.95(\mathrm{~m}, 1 \mathrm{H})$, $3.78(\mathrm{~s}, 3 \mathrm{H}), 3.73(\mathrm{~d}, \mathrm{~J}=6.9 \mathrm{~Hz}, 2 \mathrm{H}), 2.11(\mathrm{~s}, 3 \mathrm{H}) ;{ }^{13} \mathrm{C} \mathrm{NMR}\left(75 \mathrm{MHz}, \mathrm{CDCl}_{3}\right) \delta$ 205.5, 159.1, 130.6, 128.4, 114.4, 79.7, 55.5, 46.2, 38.4, 30.4; LRMS (CI) m/z calcd for $\left[\mathrm{M}+\mathrm{NH}_{4}\right] 255$, found 255.

$\mathrm{O}_{2} \mathrm{~N} \mathrm{CH}_{3}$ Prepared from trans-4-methyl- $\beta$-nitrostyrene $(163 \mathrm{mg}, 1.0 \mathrm{mmol})$ and acetone $(5.0 \mathrm{mmol}, 368 \mu \mathrm{L})$ according to General Procedure B, affording $192 \mathrm{mg}(87 \%$ yield) of white solid after chromatography $\left(4: 1\right.$, hexanes:EtOAc). $[\alpha]_{\mathrm{D}}{ }^{20}+5.5^{\circ}(\mathrm{c}$ Me $\left.\quad 1.59, \mathrm{CHCl}_{3}\right)$. IR (KBr) 3056, 3029, 2976, 2944, 2924, 1717, 1551, 1378, 1365, 1163, 816, $544 \mathrm{~cm}^{-1} ;{ }^{1} \mathrm{H}$ NMR $\left(400 \mathrm{MHz}, \mathrm{CDCl}_{3}\right) \delta$ 7.14-7.08 (m, 1H), $4.69(\mathrm{dd}, \mathrm{J}=6.9,12.3$ $\mathrm{Hz}, 1 \mathrm{H}), 4.60(\mathrm{dd}, \mathrm{J}=7.7,12.1 \mathrm{~Hz}, 1 \mathrm{H}), 4.0-3.93(\mathrm{~m}, 1 \mathrm{H}), 2.91(\mathrm{~d}, \mathrm{~J}=6.9 \mathrm{~Hz}, 2 \mathrm{H}), 2.31(\mathrm{~s}, 3 \mathrm{H})$, $2.11(\mathrm{~s}, 3 \mathrm{H}) ;{ }^{13} \mathrm{C} \mathrm{NMR}\left(75 \mathrm{MHz}, \mathrm{CDCl}_{3}\right) \delta 205.4,137.4,135.7,129.6,127.1,79.5,46.0,38.5$, 30.2, 20.9; LRMS (APCI) m/z calcd for $\mathrm{C}_{12} \mathrm{H}_{15} \mathrm{NO}_{3} 221.1$, found 221.4.

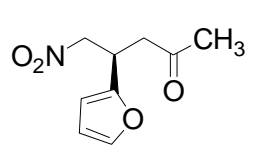

Prepared from 2-(2-nitrovinyl)furan $(139 \mathrm{mg}, 1.0 \mathrm{mmol})$ and acetone $(5.0 \mathrm{mmol}$, $368 \mu \mathrm{L})$ according to General Procedure B, affording $175 \mathrm{mg}(89 \%$ yield) of white solid after chromatography $\left(4: 1\right.$, hexanes:EtOAc). $[\alpha]_{\mathrm{D}}^{20}+5.7^{\circ}$ (c 1.32 , $\mathrm{CHCl}_{3}$ ); mp 54.5-56 ${ }^{\circ} \mathrm{C}$; IR (film) 3151, 3124, 2920, 1714, 1552, 1430, 1377, $1165,1015,741 \mathrm{~cm}^{-1} ;{ }^{1} \mathrm{H}$ NMR $\left(400 \mathrm{MHz}, \mathrm{CDCl}_{3}\right) \delta 7.34(\mathrm{dd}, \mathrm{J}=0.73,1.8 \mathrm{~Hz}, 1 \mathrm{H}), 6.30(\mathrm{dd}, \mathrm{J}$ $=1.8,5.7 \mathrm{~Hz}, 2 \mathrm{H}), 6.15(\mathrm{~d}, \mathrm{~J}=6.2 \mathrm{~Hz}, 1 \mathrm{H}), 4.72(\mathrm{dd}, \mathrm{J}=6.2,10.6 \mathrm{~Hz}, 1 \mathrm{H}), 4.67(\mathrm{dd}, \mathrm{J}=3.3,9.9$ $\mathrm{Hz}, 1 \mathrm{H}), 4.14-4.07(\mathrm{~m}, 1 \mathrm{H}), 3.01(\mathrm{dd}, \mathrm{J}=6.6,18.1 \mathrm{~Hz}, 1 \mathrm{H}), 2.93(\mathrm{dd}, \mathrm{J}=7.3,13.2 \mathrm{~Hz}, 1 \mathrm{H}), 2.18$ 
(s, 3H); ${ }^{13} \mathrm{C}$ NMR $\left(75 \mathrm{MHz}, \mathrm{CDCl}_{3}\right) \delta 205.0,151.6,142.3,110.5,107.0,77.0,43.4,32.8,30.2$; LRMS (CI) $\mathrm{m} / \mathrm{z}$ calcd for $\left[\mathrm{M}+\mathrm{NH}_{4}\right] 215$, found 215.

$\mathrm{O}_{2} \mathrm{~N} \mathrm{CH}_{3}$ Prepared from trans-2-(2-nitrovinyl)thiophene (155 mg, $\left.1.0 \mathrm{mmol}\right)$ and acetone $(5.0 \mathrm{mmol}, 368 \mu \mathrm{L})$ according to General Procedure B, affording $201 \mathrm{mg}(94 \%$ yield) of pale yellow oil after chromatography $\left(4: 1\right.$, hexanes:EtOAc). $[\alpha]_{\mathrm{D}}{ }^{20}$ $11.8^{\circ}$ (c 1.76, $\mathrm{CHCl}_{3}$ ). IR (film) 3111, 3005, 2919, 1714, 1552, 1429, 1378, 1166, 850, $707 \mathrm{~cm}^{-1}$; ${ }^{1} \mathrm{H}$ NMR $\left(400 \mathrm{MHz}, \mathrm{CDCl}_{3}\right) \delta 7.21(\mathrm{dd}, \mathrm{J}=1.1,4.8 \mathrm{~Hz}, 1 \mathrm{H}), 6.95-6.90(\mathrm{~m}, 2 \mathrm{H}), 6.15(\mathrm{~d}, \mathrm{~J}=6.2$ $\mathrm{Hz}, 1 \mathrm{H}), 4.74(\mathrm{dd}, \mathrm{J}=6.6,12.4 \mathrm{~Hz}, 1 \mathrm{H}), 4.67(\mathrm{dd}, \mathrm{J}=7.3,12.4 \mathrm{~Hz}, 1 \mathrm{H}), 4.35-4.29(\mathrm{~m}, 1 \mathrm{H}), 3.02$ $(\mathrm{d}, \mathrm{J}=6.6 \mathrm{~Hz}, 1 \mathrm{H}), 2.16(\mathrm{~s}, 3 \mathrm{H}) ;{ }^{13} \mathrm{C}$ NMR $\left(75 \mathrm{MHz}, \mathrm{CDCl}_{3}\right) \delta 205.0,141.6,127.1,125.4$, 124.6, 79.6, 46.7, 34.4, 30.2; LRMS (CI) m/z calcd for $\mathrm{C}_{9} \mathrm{H}_{11} \mathrm{NO}_{3} \mathrm{~S} 213$, found 213.

$\mathrm{O}_{2} \mathrm{~N} \mathrm{CH}_{3}$ Prepared from trans-1-nitropropene $(44 \mathrm{mg}, 0.5 \mathrm{mmol})$ and acetone $(5.0 \mathrm{mmol}$, $368 \mu \mathrm{L})$ using $15 \mathrm{~mol} \%$ of catalyst $(0.075 \mathrm{mmol})$ and $2 \mathrm{~mol} \%$ of benzoic acid ( $0.01 \mathrm{mmol})$ according to General Procedure B, affording $55 \mathrm{mg}$ ( $70 \%$ yield) of pale yellow oil after chromatography $\left(4: 1\right.$, hexanes:EtOAc). $[\alpha]_{\mathrm{D}}{ }^{20}-4.8^{\circ}\left(\mathrm{c} 1.0, \mathrm{CHCl}_{3}\right)$. IR (film) 2972, 2938, 1716, 1551, 1517, 1369, $1172 \mathrm{~cm}^{-1}$; ${ }^{1} \mathrm{H}$ NMR (400 MHz, $\left.\mathrm{CDCl}_{3}\right) \delta 4.44$ (dd, J $=6.2,11.7 \mathrm{~Hz}, 1 \mathrm{H}), 4.37(\mathrm{dd}, \mathrm{J}=6.6,11.9 \mathrm{~Hz}, 1 \mathrm{H}), 2.83(\mathrm{~m}, 1 \mathrm{H}), 2.81(\mathrm{dd}, \mathrm{J}=6.3,13.1 \mathrm{~Hz}$, $1 \mathrm{H}), 2.64(\mathrm{dd}, \mathrm{J}=6.6,17.9 \mathrm{~Hz}, 1 \mathrm{H}), 2.17(\mathrm{~s}, 3 \mathrm{H}), 1.07(\mathrm{~d}, \mathrm{~J}=6.9 \mathrm{~Hz}, 3 \mathrm{H}) ;{ }^{13} \mathrm{C}$ NMR $(75 \mathrm{MHz}$, $\left.\mathrm{CDCl}_{3}\right) \delta 206.3,80.1,46.4,30.3,28.1,17.3$; LRMS (CI) $\mathrm{m} / \mathrm{z}$ calcd for $\left[\mathrm{M}+\mathrm{NH}_{4}\right] 163$, found 163.

$\mathrm{O}_{2} \mathrm{~N} \mathrm{CH}_{3}$ Prepared from trans-4-methyl-1-nitro-pent-1-ene $72 \mathrm{mg}, 0.5 \mathrm{mmol}$ ) and acetone $(5.0 \mathrm{mmol}, 368 \mu \mathrm{L})$ using $15 \mathrm{~mol} \%$ of catalyst $(0.075 \mathrm{mmol})$ and $2 \mathrm{~mol} \%$ of benzoic acid $(0.01 \mathrm{mmol})$ according to General Procedure B, affording $82 \mathrm{mg}$ (81\% yield) of pale yellow oil after chromatography (4:1, hexanes:EtOAc). $[\alpha]_{\mathrm{D}}{ }^{20}-9.0^{\circ}$ (c 1.0 , $\mathrm{CHCl}_{3}$ ). IR (film) 2961, 2934, 2872, 1717, 1551, 1369, $735 \mathrm{~cm}^{-1} ;{ }^{1} \mathrm{H}$ NMR $\left(400 \mathrm{MHz}, \mathrm{CDCl}_{3}\right) \delta$ $4.48(\mathrm{~m}, 2 \mathrm{H}), 2.71-2.55(\mathrm{~m}, 2 \mathrm{H}), 2.54(\mathrm{dd}, \mathrm{J}=4.8,17.6 \mathrm{~Hz}, 1 \mathrm{H}), 2.17(\mathrm{~s}, 3 \mathrm{H}), 1.68-1.58(\mathrm{~m}, 1 \mathrm{H})$, 1.30-1.17 (m, 2H), $0.93(\mathrm{~d}, \mathrm{~J}=6.6 \mathrm{~Hz}, 3 \mathrm{H}), 0.91(\mathrm{~d}, \mathrm{~J}=6.6 \mathrm{~Hz}, 3 \mathrm{H}) ;{ }^{13} \mathrm{C}$ NMR $(75 \mathrm{MHz}$, $\left.\mathrm{CDCl}_{3}\right) \delta 206.7,78.5,44.7,40.5,30.8,30.4,25.0,22.4,22.3$; LRMS (CI) $\mathrm{m} / \mathrm{z}$ calcd for $[\mathrm{M}+$ $\left.\mathrm{NH}_{4}\right]$ 205, found 205 .

$\mathrm{O}_{2} \mathrm{~N} \quad \mathrm{CH}_{3}$ Prepared from trans-1-nitro-hex-1-ene $(72 \mathrm{mg}, 0.5 \mathrm{mmol})$ and acetone $(5.0$ mmol, $368 \mu \mathrm{L})$ using $15 \mathrm{~mol} \%$ of catalyst $(0.075 \mathrm{mmol})$ and $2 \mathrm{~mol} \%$ of benzoic acid $(0.01 \mathrm{mmol})$ according to General Procedure B, affording $78 \mathrm{mg}(78 \%$ yield) of pale yellow oil after chromatography $\left(4: 1\right.$, hexanes:EtOAc). $[\alpha]_{\mathrm{D}}{ }^{20}-5.0^{\circ}$ (c 0.8 , $\mathrm{CHCl}_{3}$ ). IR (film) 2934, 2864, 1719, 1551, 1435, 1379, 1167, $733 \mathrm{~cm}^{-1} ;{ }^{1} \mathrm{H}$ NMR (400 MHz, $\left.\mathrm{CDCl}_{3}\right) \delta 4.45(\mathrm{~d}, \mathrm{~J}=5.1 \mathrm{~Hz}, 2 \mathrm{H}), 2.65-2.50(\mathrm{~m}, 3 \mathrm{H}), 2.17(\mathrm{~s}, 3 \mathrm{H}), 1.41-1.29(\mathrm{~m}, 5 \mathrm{H}), 0.91(\mathrm{~m}$, $4 \mathrm{H}) ;{ }^{13} \mathrm{C}$ NMR $\left(75 \mathrm{MHz}, \mathrm{CDCl}_{3}\right) \delta 206.7,78.3,44.6,32.9,31.1,30.4,28.7,22.5,13.8$; LRMS $\left(\mathrm{CI} / \mathrm{NH}_{3}\right) \mathrm{m} / \mathrm{z}$ calcd for $\left[\mathrm{M}+\mathrm{NH}_{4}\right] 205$, found 205 .

$\begin{array}{ll}\mathrm{O}_{2} \mathrm{~N} \mathrm{CH}_{3} & \begin{array}{l}\text { Prepared from trans- } \beta \text {-nitrostyrene }(150 \mathrm{mg}, 1.0 \mathrm{mmol}) \text { and } \alpha \text {-methoxyacetone } \\ \text { (1.1 mmol, } 101 \mu \mathrm{L}) \text { according to General Procedure A, affording } 178 \mathrm{mg}(75 \%\end{array} \\ \text { yield) of white solid after chromatography }(4: 1, \text { hexanes:EtOAc). IR }(\mathrm{KBr}) 3066 \text {, }\end{array}$ $3031,3010,2933,2831,1715,1554,1380,1226,1196,1106,764,703,653,534 \mathrm{~cm}^{-1} ;{ }^{1} \mathrm{H}$ NMR $\left(400 \mathrm{MHz}, \mathrm{CDCl}_{3}\right) \delta$ 7.36-7.30 (m, 3H), $7.23(\mathrm{~m}, 2 \mathrm{H}), 4.89(\mathrm{dd}, \mathrm{J}=4.8,12.7 \mathrm{~Hz}, 1 \mathrm{H}), 4.74(\mathrm{dd}$, $\mathrm{J}=8.4,13.0 \mathrm{~Hz}, 1 \mathrm{H}), 3.90-80(\mathrm{~m}, 2 \mathrm{H}), 3.38(\mathrm{~s}, 3 \mathrm{H}), 2.04(\mathrm{~s}, 3 \mathrm{H}) ;{ }^{13} \mathrm{C} \mathrm{NMR}\left(75 \mathrm{MHz}, \mathrm{CDCl}_{3}\right) \delta$ 
208.1, 135.3, 129.1, 128.5, 128.1, 88.1, 76.7, 58.8, 45.9, 26.2; LRMS (CI) m/z calcd for $[\mathrm{M}+$ $\left.\mathrm{NH}_{4}\right]$ 255, found 255 .

On $\overbrace{\mathrm{Ph}}^{\mathrm{Me}} \mathrm{CH}_{3} \begin{aligned} & \text { Prepared from trans- } \beta \text {-nitrostyrene }(0.5 \mathrm{mmol}, 75 \mathrm{mg}) \text { and 3-pentanone }(0.75 \\ & (0.01 \mathrm{mmol}, 1.2 \mathrm{mg}) \text {, affording } 76 \mathrm{mg}(65 \% \text { yield }) \text { of white solid after }\end{aligned}$ chromatography (4:1, hexanes:EtOAc). $[\alpha]_{\mathrm{D}}^{20}+13.4^{\circ}\left(\mathrm{c} 1.55, \mathrm{CHCl}_{3}\right)$. IR (KBr) 3062, 3032, 2972, 2940, 2882, 1716, 1544, 1457, 1436, 1382, 759, $703 \mathrm{~cm}^{-1} ;{ }^{1} \mathrm{H}$ NMR $\left(400 \mathrm{MHz}, \mathrm{CDCl}_{3}\right) \delta$ 7.32-7.23 (m, 3H), 7.19-7.16 (m, 2H), $4.82(\mathrm{dd}, \mathrm{J}=5.1,12.8 \mathrm{~Hz}, 1 \mathrm{H}), 4.74(\mathrm{dd}, \mathrm{J}=9.8,12.8 \mathrm{~Hz}$, $1 \mathrm{H}), 3.81-3.75(\mathrm{~m}, 1 \mathrm{H}), 3.03-2.9(\mathrm{~m}, 1 \mathrm{H}), 2.37-2.27(\mathrm{~m}, 1 \mathrm{H}), 2.19-2.04(\mathrm{~m}, 1 \mathrm{H}), 1.20(\mathrm{~d}, \mathrm{~J}=$ $6.95 \mathrm{~Hz}, 3 \mathrm{H}), 0.85(\mathrm{t}, \mathrm{J}=7.32 \mathrm{~Hz}, 3 \mathrm{H}) ;{ }^{13} \mathrm{C} \mathrm{NMR}\left(75 \mathrm{MHz}, \mathrm{CDCl}_{3}\right) \delta 212.5,138.0,128.8,77.5$, 49.0, 45.8, 35.7, 14.3, 7.2; LRMS (APCI) $\mathrm{m} / \mathrm{z}$ calcd for $\mathrm{C}_{13} \mathrm{H}_{17} \mathrm{NO}_{3} 235.1$, found 235.2.

$\begin{array}{cl}\mathrm{OMe}_{\mathrm{C}} & \begin{array}{l}\text { Prepared from trans-4-methyl-1-nitro-pent-1-ene }(65 \mathrm{mg}, 0.5 \mathrm{mmol}) \text { and } \alpha \text { - } \\ \text { methoxyacetone }(2.0 \mathrm{mmol}, 92 \mu \mathrm{L}) \text { General Procedure } \mathrm{C} \text {, affording } 94 \mathrm{mg}(78 \%\end{array} \\ & \begin{array}{l}\text { yield) of colorless oil after chromatography }(9: 1, \text { hexanes:EtOAc).IR (film) } \\ \text { 2960, 2935, 2874, 1720,1554, 1468, 1382, 1356, 1110, 756, } 740 \mathrm{~cm}^{-1} ;{ }^{1} \mathrm{H} \text { NMR }\end{array}\end{array}$ $\left(400 \mathrm{MHz}, \mathrm{CDCl}_{3}\right) \delta 4.45(\mathrm{dd}, \mathrm{J}=5.9,13.2,1 \mathrm{H}), 4.37(\mathrm{dd}, \mathrm{J}=6.2,13.2,1 \mathrm{H}), 3.59(\mathrm{~d}, \mathrm{~J}=5.5$ $\mathrm{Hz}, 1 \mathrm{H}), 3.38(\mathrm{~s}, 3 \mathrm{H}), 2.69-2.63(\mathrm{~m}, 1 \mathrm{H}), 2.21(\mathrm{~s}, 3 \mathrm{H}), 1.68-1.58(\mathrm{~m}, 1 \mathrm{H}), 1.68-1.60(\mathrm{~m}, 1 \mathrm{H})$, 1.34-1.17 (m, 2H), $0.93(\mathrm{~d}, \mathrm{~J}=2.9 \mathrm{~Hz}, 3 \mathrm{H}), 0.92(\mathrm{~d}, \mathrm{~J}=3.3 \mathrm{~Hz}, 3 \mathrm{H}) ;{ }^{13} \mathrm{C}$ NMR $(75 \mathrm{MHz}$, $\left.\mathrm{CDCl}_{3}\right) \delta 209.4,87.0,75.6,58.9,37.4,37.3,26.1,22.5,22.1 ;$ LRMS (CI) $\mathrm{m} / \mathrm{z}$ calcd for $[\mathrm{M}+$ $\left.\mathrm{NH}_{4}\right]$ 235, found 235 .

$\mathrm{O}_{2} \mathrm{~N}$ Prepared from trans- $\beta$-nitrostyrene $(0.5 \mathrm{mmol}, 75 \mathrm{mg})$ and 4-methyl-pent-2one $(1.1 \mathrm{mmol}, 69 \mu \mathrm{L})$ according to General Procedure A, affording $88 \mathrm{mg}$ (71\% yield) of white solid after chromatography $\left(4: 1\right.$, hexanes:EtOAc). $[\alpha]_{\mathrm{D}}{ }^{20}$

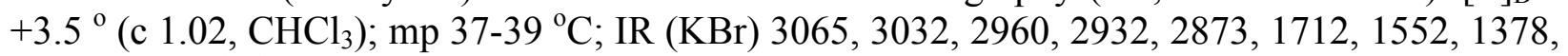
$761,701 \mathrm{~cm}^{-1}$; ${ }^{1} \mathrm{H}$ NMR $\left(400 \mathrm{MHz}, \mathrm{CDCl}_{3}\right) \delta$ 7.35-7.20 (m, 5H), $4.72(\mathrm{dd}, \mathrm{J}=7.0,12.4 \mathrm{~Hz}, 1 \mathrm{H})$, $4.63(\mathrm{dd}, \mathrm{J}=7.7,12.5 \mathrm{~Hz}, 1 \mathrm{H}), 4.05(\mathrm{~m}, 1 \mathrm{H}), 2.91(\mathrm{abq}, \mathrm{J}=6.6,15.4 \mathrm{~Hz}, 2 \mathrm{H}), 2.28(\mathrm{abq}, \mathrm{J}=7.3$, $13.9 \mathrm{~Hz}, 2 \mathrm{H}), 2.12(\mathrm{~m}, 1 \mathrm{H}), 0.85(\mathrm{~d}, \mathrm{~J}=6.3 \mathrm{~Hz}, 3 \mathrm{H}), 0.84(\mathrm{~d}, \mathrm{~J}=6.3 \mathrm{~Hz}, 3 \mathrm{H}) ;{ }^{13} \mathrm{C}$ NMR $(75$ $\left.\mathrm{MHz}, \mathrm{CDCl}_{3}\right) \delta 207.5,138.9,129.0,127.8,127.4,79.4,52.2,45.7$ 39.0, 24.4, 22.4, 22.3,; LRMS (CI) $\mathrm{m} / \mathrm{z}$ calcd for $\left[\mathrm{M}+\mathrm{NH}_{4}\right] 267$, found 267 .

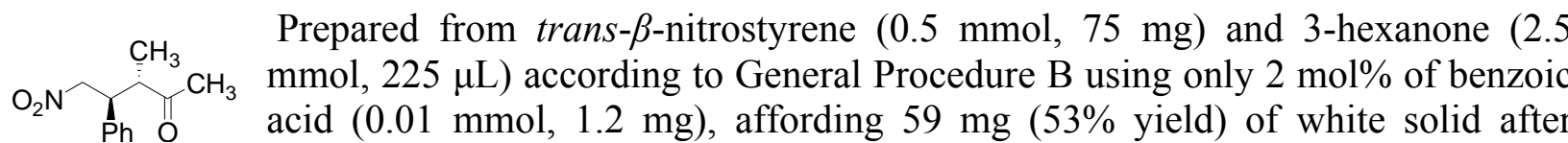
chromatography $\left(4: 1\right.$, hexanes:EtOAc). $[\alpha]_{\mathrm{D}}{ }^{20}-4.8^{\circ}\left(\mathrm{c} 0.8, \mathrm{CHCl}_{3}\right)$. IR $(\mathrm{KBr})$ $3084,3064,3035,2986,1710,1544,1437,1385,1282,1170,1089,759,703,536 \mathrm{~cm}^{-1} ;{ }^{1} \mathrm{H}$ NMR (400 MHz, $\left.\mathrm{CDCl}_{3}\right) \delta$ 7.33-7.19 (m, 5H), $4.80(\mathrm{dd}, \mathrm{J}=4.9,12.7 \mathrm{~Hz}, 1 \mathrm{H}), 4.73(\mathrm{dd}, \mathrm{J}=9.3$, $12.9 \mathrm{~Hz}, 1 \mathrm{H}), 3.81(\mathrm{~m}, 1 \mathrm{H}), 3.02(\mathrm{~m}, 1 \mathrm{H}), 1.95(\mathrm{~s}, 3 \mathrm{H}) ; 1.23(\mathrm{~d}, \mathrm{~J}=7.3,3 \mathrm{H}) ;{ }^{13} \mathrm{C}$ NMR $(75 \mathrm{MHz}$, $\left.\mathrm{CDCl}_{3}\right) \delta 209.8,137.9,128.9,127.9,127.8,77.5,49.7,45.7,29.4,14.2$; LRMS (ES) m/z calcd for $\left[\mathrm{M}+\mathrm{NH}_{4}\right] 239$, found 239 .

$\mathrm{O}_{2} \mathrm{~N} \quad \mathrm{CH}_{3}$ Prepared from trans- $\beta$-nitrostyrene $(0.5 \mathrm{mmol}, 75 \mathrm{mg})$ and 3-hexanone $(2.5$ $\mathrm{Ph} O \mathrm{CH}_{3} \mathrm{mmol}, 225 \mu \mathrm{L}$ ) according to General Procedure B using only $2 \mathrm{~mol} \%$ of benzoic acid $(0.01 \mathrm{mmol}, 1.2 \mathrm{mg})$, affording $28 \mathrm{mg}(25 \%$ yield $)$ of white solid after chromatography $\left(4: 1\right.$, hexanes:EtOAc). $[\alpha]_{\mathrm{D}}{ }^{20}+7.06^{\circ}\left(\mathrm{c} 0.75, \mathrm{CHCl}_{3}\right)$. IR $(\mathrm{KBr}) 3085,3062$, 3032, 2986, 2949, 2906, 1718, 1548, 1379, 1120, 762, 697, 571, $543 \mathrm{~cm}^{-1} ;{ }^{1} \mathrm{H}$ NMR (400 MHz, 
$\left.\mathrm{CDCl}_{3}\right) \delta 7.35-7.20(\mathrm{~m}, 5 \mathrm{H}), 4.72(\mathrm{dd}, \mathrm{J}=7.0,12.4 \mathrm{~Hz}, 1 \mathrm{H}), 4.63(\mathrm{dd}, \mathrm{J}=7.7,12.1 \mathrm{~Hz}, 1 \mathrm{H})$, $4.06(\mathrm{~m}, 1 \mathrm{H}), 2.89(\mathrm{~d}, \mathrm{~J}=7.0,2 \mathrm{H}), 2.462 .24(\mathrm{~m}, 2 \mathrm{H}), 1.02(\mathrm{t}, \mathrm{J}=7.3,3 \mathrm{H}) ;{ }^{13} \mathrm{C} \mathrm{NMR}(75 \mathrm{MHz}$, $\left.\mathrm{CDCl}_{3}\right) \delta 208.1,138.9,129.0,127.8,127.4,79.5,44.9,39.1,36.5,7.5$; LRMS (ES) $\mathrm{m} / \mathrm{z}$ calcd for $\left[\mathrm{M}+\mathrm{NH}_{4}\right] 239$, found 239 .

$\mathrm{O}_{2} \mathrm{~N}$ Prepared from trans- $\beta$-nitrostyrene $(0.5 \mathrm{mmol}, 75 \mathrm{mg})$ and 3-hexanone $(2.0$ mmol, $110 \mu \mathrm{L}$ ) according to General Procedure A affording 78mg (67\% yield) of colorless oil after chromatography $\left(4: 1\right.$, hexanes:EtOAc). $[\alpha]_{\mathrm{D}}{ }^{20}+6.0^{\circ}$ (c $\left.0.58, \mathrm{CHCl}_{3}\right)$. IR (KBr) 3065, 3033, 2965, 2935, 2877, 1712, 1551, 1378, 764, $701 \mathrm{~cm}^{-1} ;{ }^{1} \mathrm{H}$ NMR $\left(400 \mathrm{MHz}, \mathrm{CDCl}_{3}\right) \delta$ 7.35-7.20 (m, 5H), $4.72(\mathrm{dd}, \mathrm{J}=6.8,12.2 \mathrm{~Hz}, 1 \mathrm{H}), 4.63(\mathrm{dd}, \mathrm{J}=7.8$, $12.2 \mathrm{~Hz}, 1 \mathrm{H}), 4.05(\mathrm{~m}, 1 \mathrm{H}), 2.92(\mathrm{abq}, \mathrm{J}=6.8,17.6 \mathrm{~Hz}, 2 \mathrm{H}), 2.39(\mathrm{~m}, 2 \mathrm{H}), 1.58(\mathrm{~m}, 2 \mathrm{H}), 0.86(\mathrm{t}$, $\mathrm{J}=7.32,3 \mathrm{H}) ;{ }^{13} \mathrm{C} \mathrm{NMR}\left(75 \mathrm{MHz}, \mathrm{CDCl}_{3}\right) \delta 207.8,138.9,129.0,127.8,127.4,79.5,45.2,45.1$, 39.0, 17.0, 13.5; LRMS (ES) m/z calcd for $\left[\mathrm{M}+\mathrm{NH}_{4}\right] 253$, found 253.

$\mathrm{CH}_{3} \quad$ Prepared from trans- $\beta$-nitrostyrene $(0.5 \mathrm{mmol}, 75 \mathrm{mg})$ and 3 -hexanone (1.5 $\mathrm{O}_{2} \mathrm{~N}$ acid $(0.01 \mathrm{mmol}, 1.2 \mathrm{mg})$, affording $64 \mathrm{mg}$ (51\% yield) of white solid after chromatography $\left(4: 1\right.$, hexanes:EtOAc). $[\alpha]_{\mathrm{D}}{ }^{20}+13.0^{\circ}$ (c $\left.0.42, \mathrm{CHCl}_{3}\right)$; mp 61.5-63.0 ${ }^{\circ} \mathrm{C}$; IR (KBr) 3064, 3032, 2966, 2880, 1714, 1542, 1434, 1384, 759, $703 \mathrm{~cm}^{-1} ;{ }^{1} \mathrm{H}$ NMR (400 MHz, $\left.\mathrm{CDCl}_{3}\right) \delta 7.32-7.17(\mathrm{~m}, 5 \mathrm{H}), 4.81(\mathrm{dd}, \mathrm{J}=5.4,12.7 \mathrm{~Hz}, 1 \mathrm{H}), 4.73(\mathrm{dd}, \mathrm{J}=9.8,12.7 \mathrm{~Hz}, 1 \mathrm{H})$, $3.81(\mathrm{~m}, 1 \mathrm{H}), 2.99(\mathrm{~m}, 1 \mathrm{H}), 2.30(\mathrm{dt}, \mathrm{J}=7.3,17.1 \mathrm{~Hz}, 1 \mathrm{H}), 2.08(\mathrm{dt}, \mathrm{J}=7.3,17.6 \mathrm{~Hz}, 1 \mathrm{H}), 1.41$ $(\mathrm{m}, 2 \mathrm{H}), 1.20(\mathrm{~d}, \mathrm{~J}=6.8 \mathrm{~Hz}, 3 \mathrm{H}), 0.72(\mathrm{t}, \mathrm{J}=7.32,3 \mathrm{H}) ;{ }^{13} \mathrm{C} \mathrm{NMR}\left(75 \mathrm{MHz}, \mathrm{CDCl}_{3}\right) \delta 211.8$, 138.0, 128.9, 127.9, 127.8, 77.6, 49.2, 45.8, 44.4, 19.5, 16.5, 14.4, 13.4; LRMS (CI) m/z calcd for $\left[\mathrm{M}+\mathrm{NH}_{4}\right] 267$, found 267 .

$\mathrm{CH}_{3} \quad$ Prepared from trans- $\beta$-nitrostyrene $(0.5 \mathrm{mmol}, 75 \mathrm{mg})$ and 3-heptanone (1.5 $\mathrm{O}_{2} \mathrm{~N}$ mmol, $105 \mu \mathrm{L}$ ) according to General Procedure B using only $2 \mathrm{~mol} \%$ of benzoic acid (0.01 mmol, $1.2 \mathrm{mg})$, affording $69 \mathrm{mg}(53 \%$ yield) of white solid after chromatography $\left(4: 1\right.$, hexanes:EtOAc). $[\alpha]_{\mathrm{D}}{ }^{20}+11.9^{\circ}\left(\mathrm{c} 0.39, \mathrm{CHCl}_{3}\right)$; mp $64-65^{\circ} \mathrm{C}$; IR (KBr) 2955, 2934, 2870, 1708, 1542, 1437, 1383, 755, $701 \mathrm{~cm}^{-1} ;{ }^{1} \mathrm{H}$ NMR $\left(400 \mathrm{MHz}, \mathrm{CDCl}_{3}\right) \delta$ 7.31-7.28 (m, 2H), 7.26-7.23 (m, 1H), $7.18(\mathrm{~d}, \mathrm{~J}=6.8 \mathrm{~Hz}, 2 \mathrm{H}), 4.81(\mathrm{dd}, \mathrm{J}=4.9,12.7 \mathrm{~Hz}, 1 \mathrm{H})$, $4.73(\mathrm{dd}, \mathrm{J}=9.8,12.7 \mathrm{~Hz}, 1 \mathrm{H}), 3.81(\mathrm{~m}, 1 \mathrm{H}), 3.01(\mathrm{~m}, 1 \mathrm{H}), 2.31(\mathrm{dt}, \mathrm{J}=7.3,17.1 \mathrm{~Hz}, 1 \mathrm{H}), 2.10$ $(\mathrm{dt}, \mathrm{J}=7.3,17.6 \mathrm{~Hz}, 1 \mathrm{H}), 1.34(\mathrm{~m}, 2 \mathrm{H}), 1.20(\mathrm{~d}, \mathrm{~J}=6.8 \mathrm{~Hz}, 3 \mathrm{H}), 1.14(\mathrm{~m}, 2 \mathrm{H}), 0.79(\mathrm{t}, \mathrm{J}=7.3$, $3 \mathrm{H}) ;{ }^{13} \mathrm{C}$ NMR $\left(75 \mathrm{MHz}, \mathrm{CDCl}_{3}\right) \delta 212.0,138.0,128.9,127.9,77.6,49.2,45.8,42.2,25.1,22.0$, 14.4, 13.4; LRMS (CI) m/z calcd for $\left[\mathrm{M}+\mathrm{NH}_{4}\right] 281$, found 281 .

$\mathrm{CH}_{3} \quad$ Prepared from trans- $\beta$-nitrostyrene $(0.5 \mathrm{mmol}, 75 \mathrm{mg})$ and 3-heptanone (1.5 $\mathrm{O}_{2} \mathrm{~N}$ mmol, $117 \mu \mathrm{L}$ ) according to General Procedure B using only $2 \mathrm{~mol} \%$ of $\mathrm{Ph} O \quad$ benzoic acid $(0.01 \mathrm{mmol}, 1.2 \mathrm{mg})$, affording $70 \mathrm{mg}$ (50\% yield) of white solid after chromatography $\left(4: 1\right.$, hexanes:EtOAc). $[\alpha]_{\mathrm{D}}{ }^{20}+13.2^{\circ}$ (c $\left.0.93, \mathrm{CHCl}_{3}\right)$; mp 61.5-63.0 ${ }^{\circ} \mathrm{C}$; IR (KBr) 3065, 3012, 2956, 2872, 1709, 1543, 1459, 1438, 1382, $700 \mathrm{~cm}^{-1}$; ${ }^{1} \mathrm{H}$ NMR (400 $\left.\mathrm{MHz}, \mathrm{CDCl}_{3}\right) \delta$ 7.31-7.28 (m, 2H), 7.26-7.23 (m, 1H), $7.19(\mathrm{~d}, \mathrm{~J}=6.8 \mathrm{~Hz}, 2 \mathrm{H}), 4.81(\mathrm{dd}, \mathrm{J}=5.4$, $12.7 \mathrm{~Hz}, 1 \mathrm{H}), 4.73(\mathrm{dd}, \mathrm{J}=9.8,12.7 \mathrm{~Hz}, 1 \mathrm{H}), 3.81(\mathrm{~m}, 1 \mathrm{H}), 3.00(\mathrm{~m}, 1 \mathrm{H}), 2.30(\mathrm{dt}, \mathrm{J}=7.3,17.1$ $\mathrm{Hz}, 1 \mathrm{H}), 2.10(\mathrm{dt}, \mathrm{J}=7.3,17.6 \mathrm{~Hz}, 1 \mathrm{H}), 1.36(\mathrm{~m}, 2 \mathrm{H}), 1.20(\mathrm{~d}, \mathrm{~J}=6.8 \mathrm{~Hz}, 3 \mathrm{H}), 1.18$ (partially ovrlp, m, 2H), $1.05(\mathrm{~m}, 2 \mathrm{H}), 0.82(\mathrm{t}, \mathrm{J}=7.3,3 \mathrm{H}) ;{ }^{13} \mathrm{C} \mathrm{NMR}\left(75 \mathrm{MHz}, \mathrm{CDCl}_{3}\right) \delta 212.0,138.0$, 128.8, 127.9, 127.8, 77.6, 49.2, 45.8, 42.5, 31.0, 22.7, 22.3, 14.4, 13.8; LRMS (CI) m/z calcd for $\left[\mathrm{M}+\mathrm{NH}_{4}\right] 295$, found 295 . 


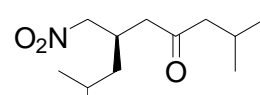

Prepared from trans-4-methyl-1-nitro-pent-1-ene $(65 \mathrm{mg}, 0.5 \mathrm{mmol})$ and 4methyl-pent-2-one $(5.0 \mathrm{mmol}, 312 \mu \mathrm{L})$ General Procedure D, affording $64 \mathrm{mg}$ (56\% yield) of colorless oil after chromatography (95:5, hexanes:EtOAc). $[\alpha]_{\mathrm{D}}{ }^{20}-8.6^{\circ}\left(\mathrm{c} 0.5, \mathrm{CHCl}_{3}\right)$. IR (KBr) 2960, 2873, 1712, 1552, 1468, 1369, $1171 \mathrm{~cm}^{-1} ;{ }^{1} \mathrm{H}$ NMR $\left(400 \mathrm{MHz}, \mathrm{CDCl}_{3}\right) \delta 4.45(\mathrm{~d}, \mathrm{~J}=5.5 \mathrm{~Hz}, 2 \mathrm{H}), 2.72(\mathrm{~m}, 1 \mathrm{H}), 2.61(\mathrm{dd}, \mathrm{J}=7.3,17.9 \mathrm{~Hz}, 1 \mathrm{H}), 2.49$ $(\mathrm{dd}, \mathrm{J}=5.5,17.9 \mathrm{~Hz}, 1 \mathrm{H}), 2.30(\mathrm{~d}, \mathrm{~J}=6.6 \mathrm{~Hz}, 2 \mathrm{H}), 2.19(\mathrm{~m}, 1 \mathrm{H}), 1.68(\mathrm{~m}, 1 \mathrm{H}), 1.33(\mathrm{~m}, 2 \mathrm{H})$, $0.93(\mathrm{~m}, 12 \mathrm{H}) ;{ }^{13} \mathrm{C}$ NMR $\left(75 \mathrm{MHz}, \mathrm{CDCl}_{3}\right) \delta 208.9,78.6,52.3,49.2,44.4,40.6,30.8,25.1,24.5$, 22.5, 22.4, 22.3; LRMS (CI) $\mathrm{m} / \mathrm{z}$ calcd for $\left[\mathrm{M}+\mathrm{NH}_{4}\right] 247$, found 247.

$\mathrm{O}_{2} \mathrm{~N}$ Prepared from trans-4-methyl-1-nitro-pent-1-ene (65 mg, $\left.0.5 \mathrm{mmol}\right)$ and 2pentanone $(5.0 \mathrm{mmol}, 270 \mu \mathrm{L})$ General Procedure D, affording $53 \mathrm{mg}(50 \%$ yield) of colorless oil after chromatography $\left(9.5: 0.5\right.$, hexanes:EtOAc). $[\alpha]_{\mathrm{D}}{ }^{20}-$ $8.3^{\circ}$ (c $\left.0.3, \mathrm{CHCl}_{3}\right)$. IR (KBr) 2962, 2935, 2875, 1713, 1551, 1381, 913, $735 \mathrm{~cm}^{-1} ;{ }^{1} \mathrm{H}$ NMR $(400$ $\left.\mathrm{MHz} \mathrm{CDCl}_{3}\right) \delta 4.4(\mathrm{~d}, \mathrm{~J}=5.9 \mathrm{~Hz}, 2 \mathrm{H}), 2.72(\mathrm{~m}, 1 \mathrm{H}), 2.61(\mathrm{dd}, \mathrm{J}=7.3,18.1 \mathrm{~Hz}, 1 \mathrm{H}), 2.50(\mathrm{dd}, \mathrm{J}$ $=5.4,18.1 \mathrm{~Hz}, 1 \mathrm{H}), 2.41(\mathrm{t}, \mathrm{J}=7.3 \mathrm{~Hz}, 2 \mathrm{H}), 1.66(\mathrm{~m}, 3 \mathrm{H}), 1.29(\mathrm{~m}, 2 \mathrm{H}), 0.93(\mathrm{~m}, 9 \mathrm{H}) ;{ }^{13} \mathrm{C} \mathrm{NMR}$ $\left(75 \mathrm{MHz}, \mathrm{CDCl}_{3}\right) \delta 209.1,78.6,45.2,43.9,40.6,30.9,25.1,22.5,22.3,17.1,13.7$; LRMS (CI) $\mathrm{m} / \mathrm{z}$ calcd for $\left[\mathrm{M}+\mathrm{NH}_{4}\right] 233$, found 233 . 
$\mathrm{AD}, 208 \mathrm{ipa} / \mathrm{hex}, 1 \mathrm{~mL} / \mathrm{min}$

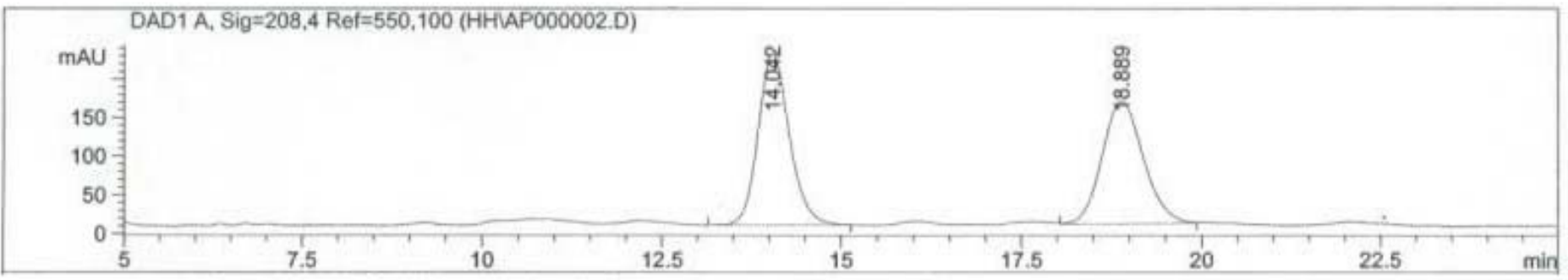

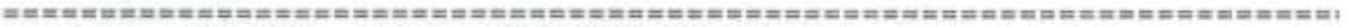

Area Percent Report

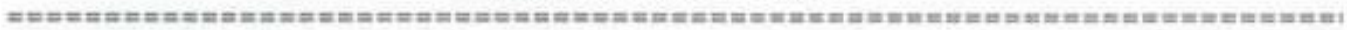

Peak RetTime Type width Area Height Area

\begin{tabular}{ll|l|l|l|l|l|}
$\#$ & {$[\mathrm{~min}]$} & [min] & [mAU*g] & \multicolumn{1}{c}{ [mAU] } & t \\
\hline 1 & 14.042 & $\mathrm{~PB}$ & 0.4463 & 6748.23437 & 222.23282 & 51.5525 \\
2 & 18.889 & $\mathrm{VV}$ & 0.5575 & 6341.78955 & 158.94890 & 48.4475
\end{tabular}

Totals :

$1.30900 \mathrm{e} 4 \quad 381.18172$
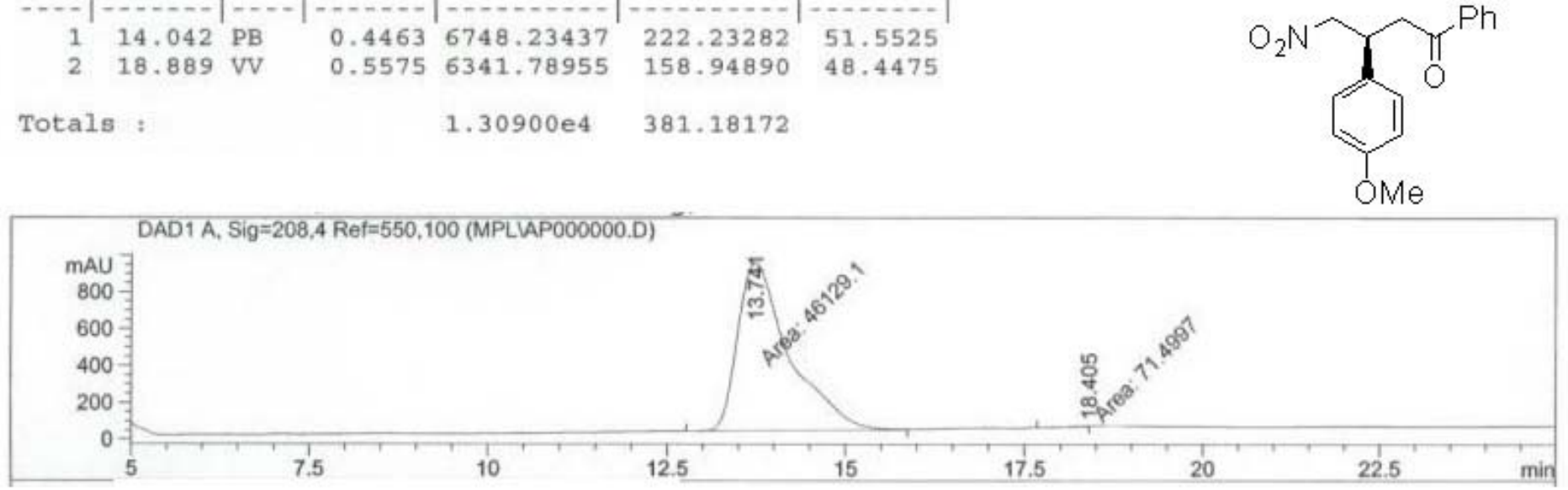

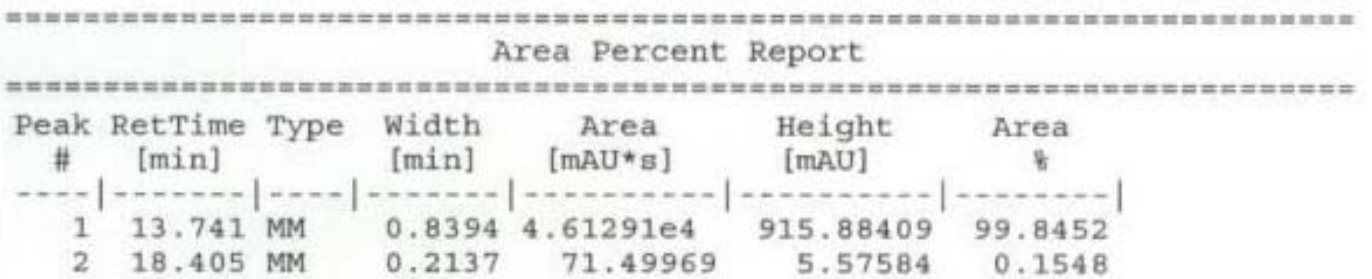

Totals :

$4.62006 \mathrm{e} 4 \quad 921.45994$ 
158 ipa/hex, $0.9 \mathrm{ml} / \mathrm{min}$ AS

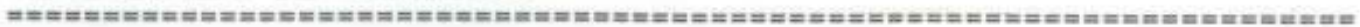
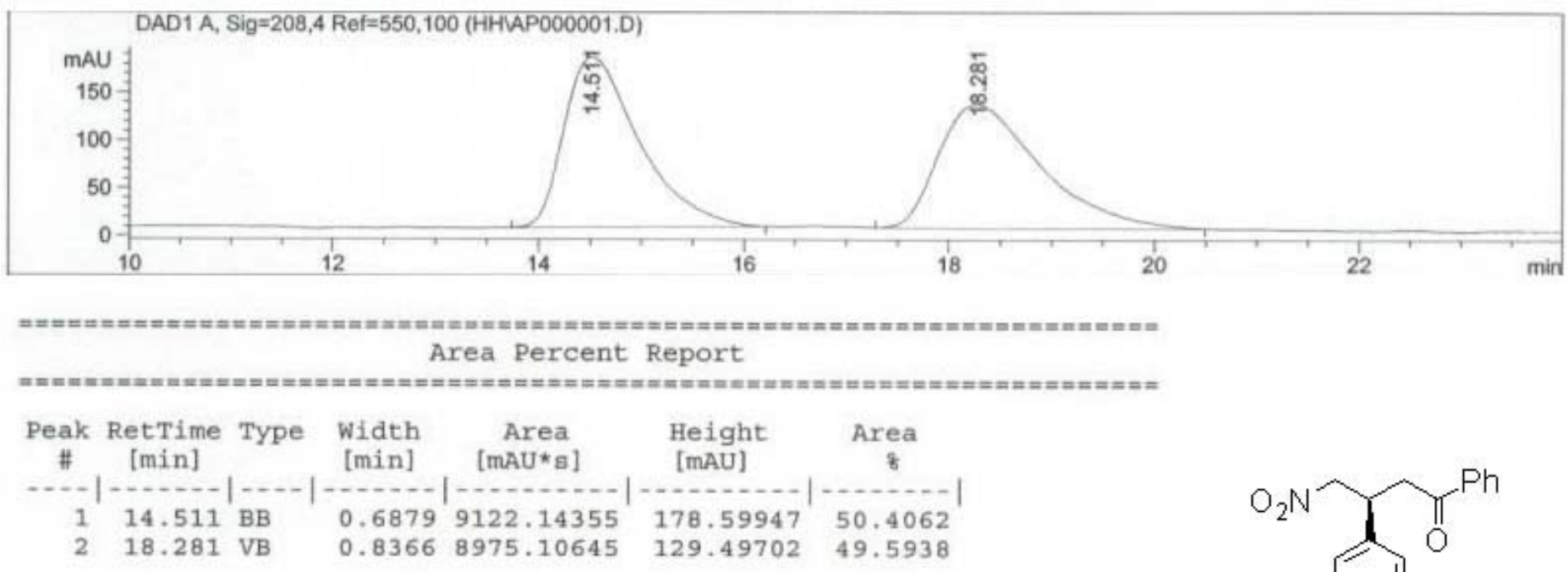

Totals :

$1.80973 \mathrm{e} \quad 308.09650$
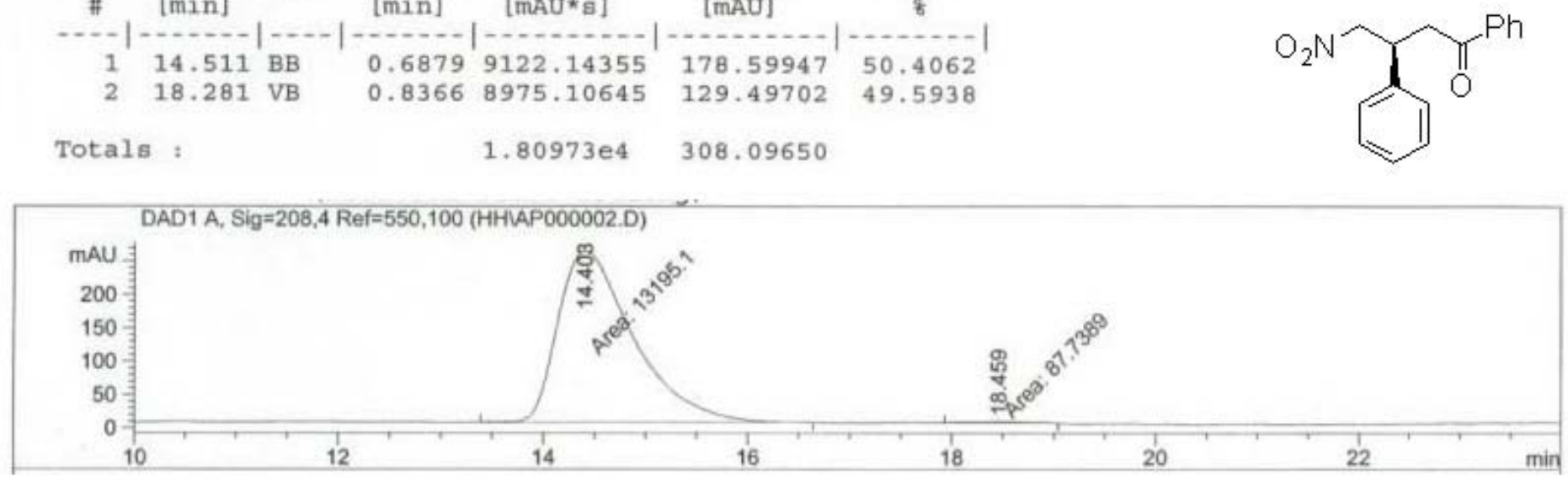

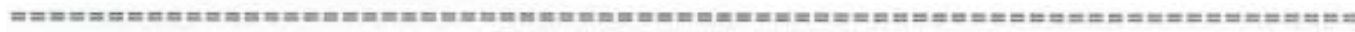

Area Percent Report

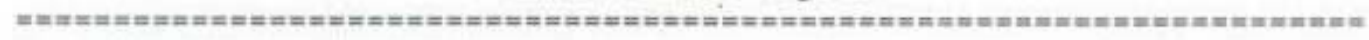

peak RetTime Type width Area Area

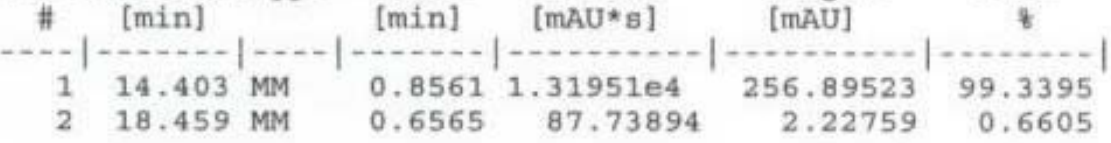

Totals :

$1.32828 \mathrm{e} \quad 259.12282$ 
25 ipa/hex, $1 \mathrm{~mL} / \mathrm{min}$, AS

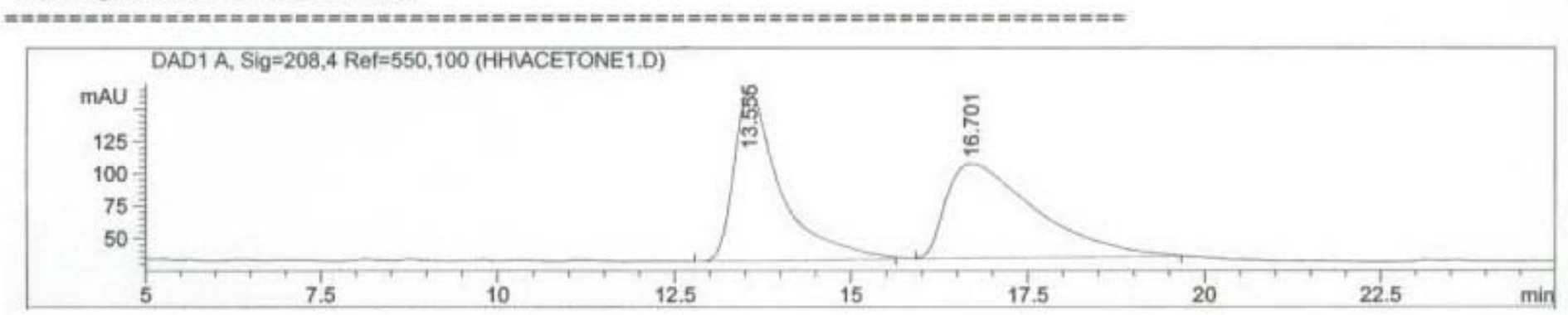

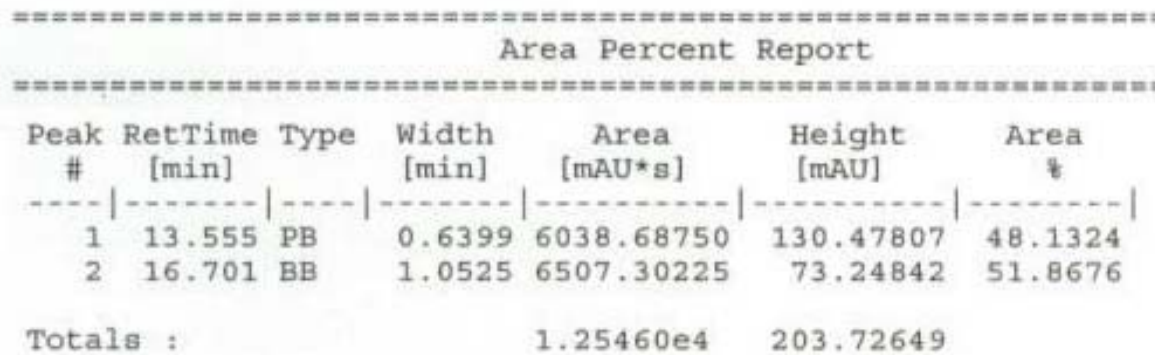

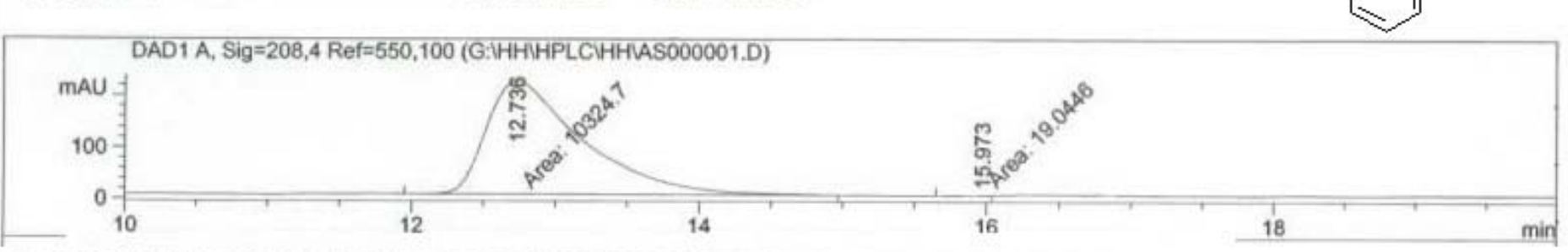

\begin{tabular}{|c|c|c|c|c|c|c|}
\hline $\begin{array}{c}\text { Peak } \\
\#\end{array}$ & $\begin{array}{l}\text { RetTime } \\
\text { [min] }\end{array}$ & Type & $\begin{array}{c}\text { Width } \\
\text { [min] }\end{array}$ & $\begin{array}{c}\text { Area } \\
{\left[m A U^{*} s\right]}\end{array}$ & $\begin{array}{l}\text { Height } \\
\text { [mAU]] }\end{array}$ & $\underset{f}{\text { Area }}$ \\
\hline & & & & & & \\
\hline $\begin{array}{l}1 \\
2\end{array}$ & 12.736 & MM & 0.7849 & 1. $03247 \mathrm{e} 4$ & 219.23091 & 99.8159 \\
\hline & 15.973 & MM & 0,3146 & 19.04464 & 907 & \\
\hline
\end{tabular}

Totals :

$$
\text { 1. } 03438 \mathrm{e} 4 \quad 220.23998
$$


OD, 8\% IPA/Hex, $0.6 \mathrm{~mL} / \mathrm{min}$
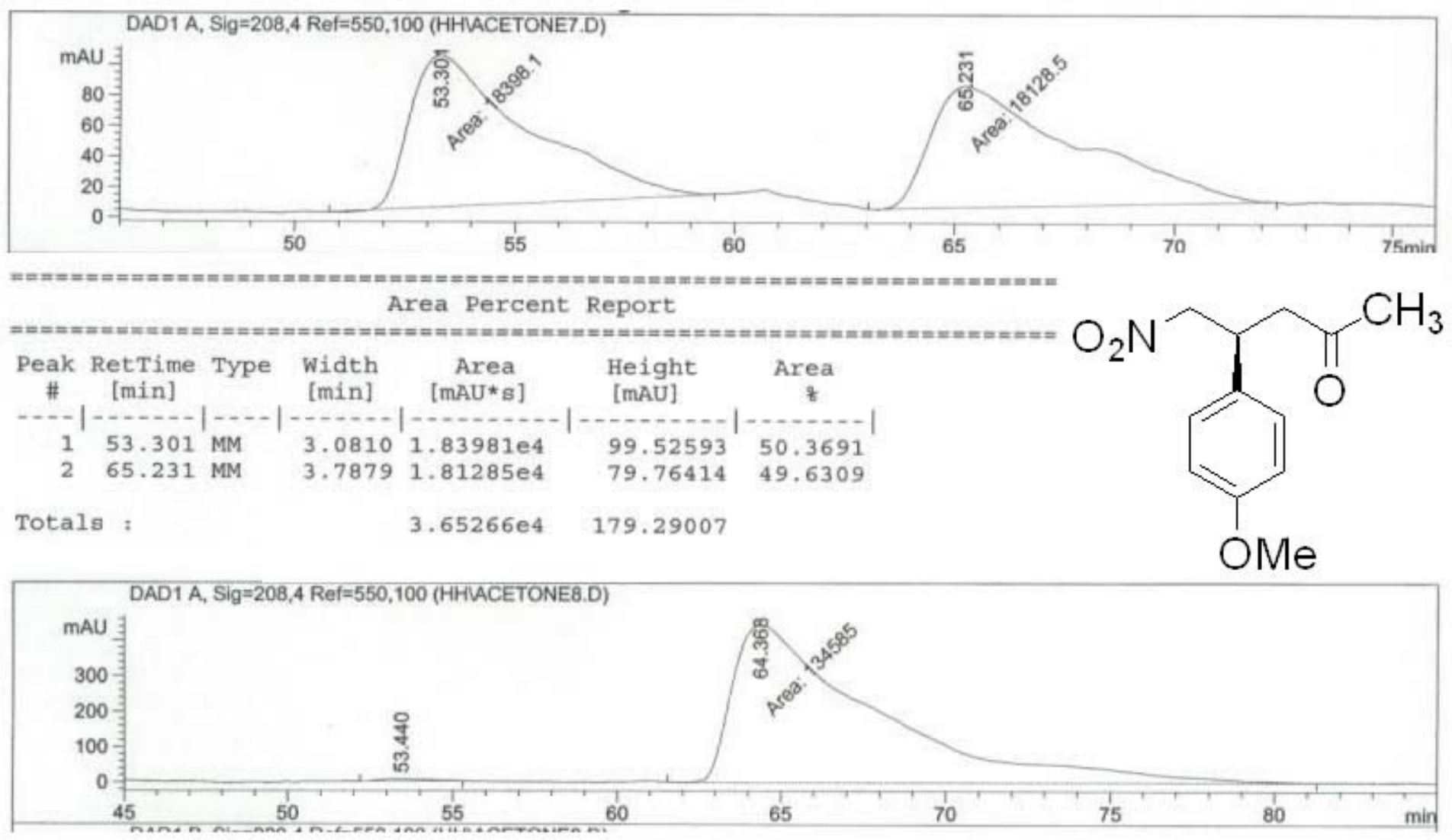

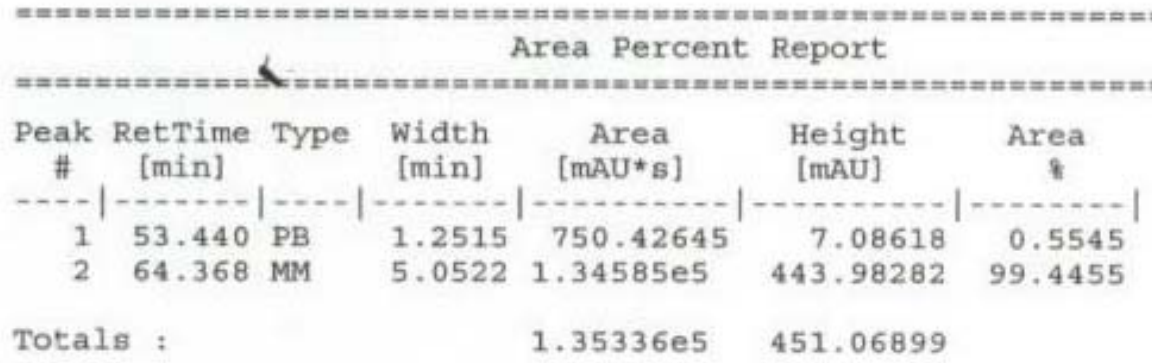


OD, $8 \%$ IPA $/$ Hex, $0.8 \mathrm{~mL} / \mathrm{min}$

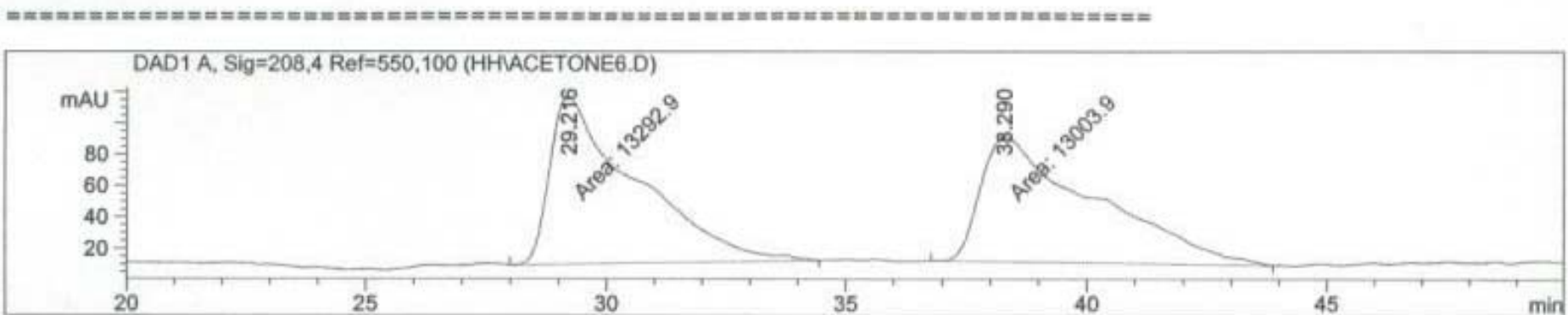

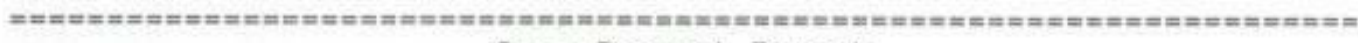

Area Percent Report

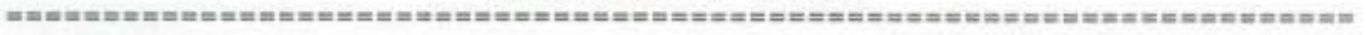

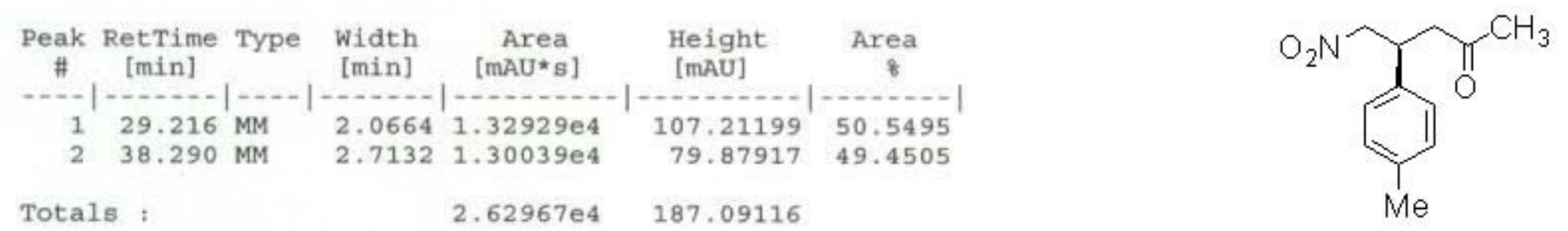

Totals :

$2.62967 \mathrm{e} 4 \quad 187.09116$

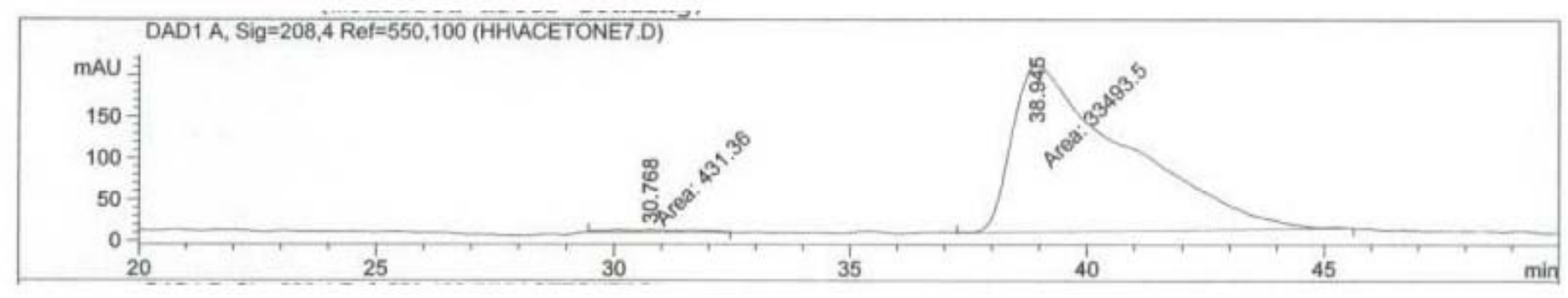

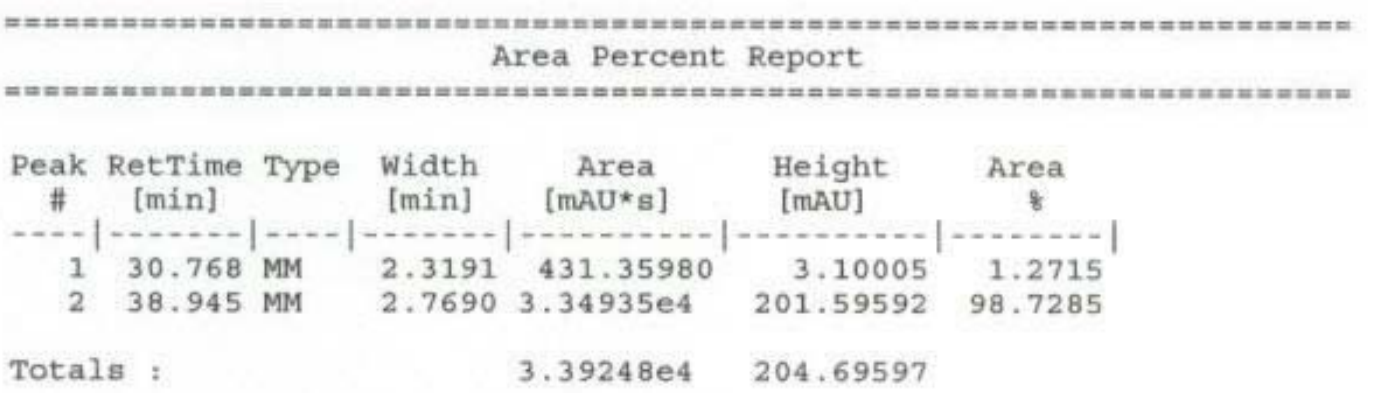


$\mathrm{AD}, 6 \% \mathrm{IPA} / \mathrm{Hex}, 0.6 \mathrm{~mL} / \mathrm{min}$

DAD1 A, Sig $=208,4$ Ref $=550,100$ (HHIACETONE3.D)

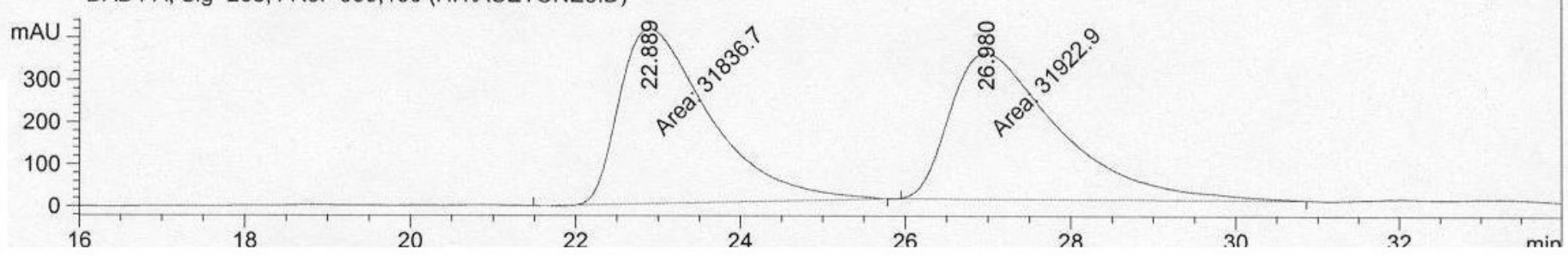

Signal 1: DAD1 A, Sig=208,4 Ref $=550,100$

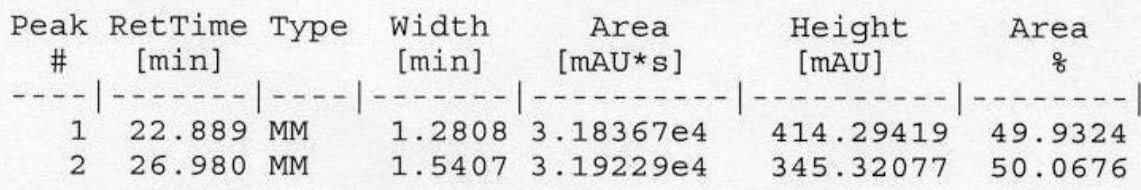

Totals :

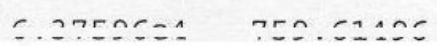
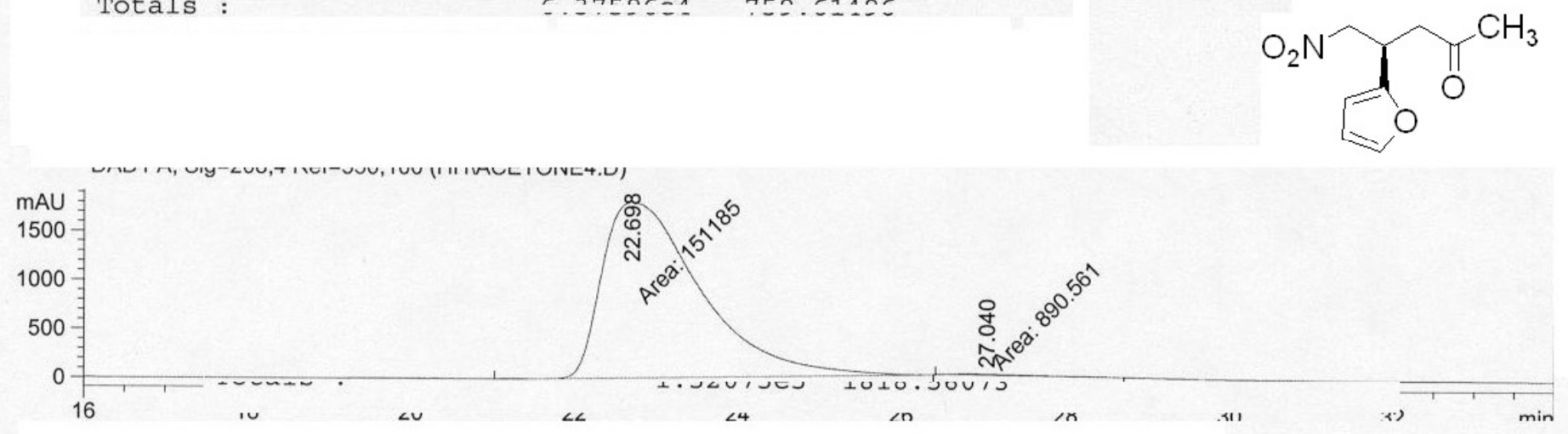

Signal 1: DAD1 A, Sig=208,4 Ref $=550,100$

\begin{tabular}{|c|c|c|c|c|c|c|}
\hline $\begin{array}{c}\text { Peak } \\
\#\end{array}$ & $\begin{array}{c}\text { RetTime } \\
\text { [min] }\end{array}$ & Type & $\begin{array}{c}\text { Width } \\
\text { [min] }\end{array}$ & $\begin{array}{c}\text { Area } \\
{\left[\mathrm{mAU}{ }^{*} \mathrm{~s}\right]}\end{array}$ & $\begin{array}{l}\text { Height } \\
\text { [mAU] }\end{array}$ & $\begin{array}{c}\text { Area } \\
\frac{\%}{\circ}\end{array}$ \\
\hline & & & & - & مص & ---- \\
\hline $\begin{array}{l}1 \\
2\end{array}$ & $\begin{array}{l}22.698 \\
27.040\end{array}$ & $\begin{array}{l}\text { MM } \\
\text { MM }\end{array}$ & $\begin{array}{l}1.3936 \\
1.4221\end{array}$ & $\begin{array}{r}1.51185 e 5 \\
890.56140\end{array}$ & $\begin{array}{r}1808.12354 \\
10.43719\end{array}$ & $\begin{array}{r}99.4144 \\
0.5856\end{array}$ \\
\hline
\end{tabular}

Totals.

1 โวก7รด5 1010 Eะก7ว 

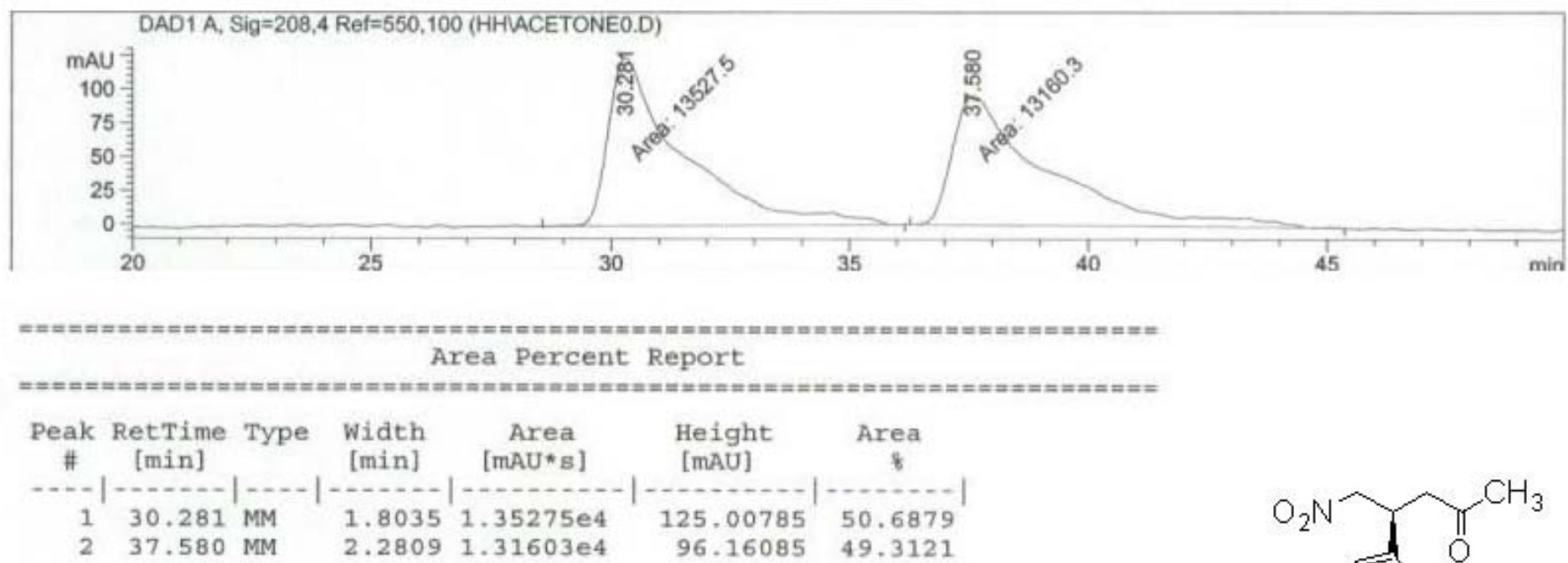

Totals :

$2.66877 \mathrm{e} 4$

221.16870

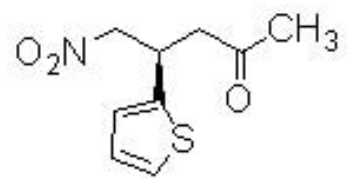

DAD1 A, Sig $=208,4$ Ref $=550,100$ (HHACETONE1,D)

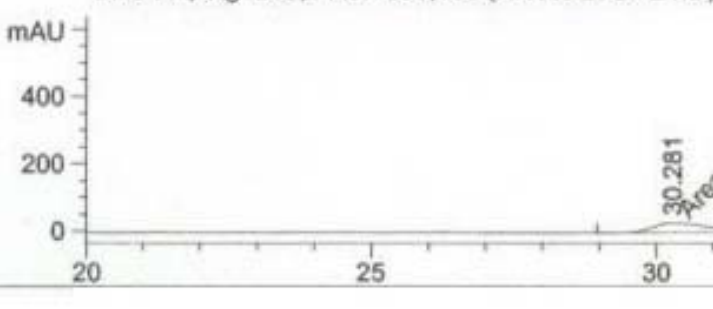

\begin{tabular}{|c|c|c|c|c|c|c|}
\hline $\begin{array}{c}\text { Peak } \\
\#\end{array}$ & $\begin{array}{l}\text { RetTime } \\
\text { [min] }\end{array}$ & Type & $\begin{array}{l}\text { Width } \\
\text { [min] }\end{array}$ & $\begin{array}{c}\text { Area } \\
\text { [mAU*s] }\end{array}$ & $\begin{array}{l}\text { Height } \\
\text { [mAU] }\end{array}$ & $\underset{t}{\text { Area }}$ \\
\hline 1 & 30,28 & MM & 1.38 & 2397.67505 & 28,93632 & 00.92 \\
\hline 2 & 37.159 & $\mathrm{MM}$ & 3.1981 & $1.16938 \mathrm{e} 5$ & 609.41809 & 97.9908 \\
\hline
\end{tabular}

Totals : $\quad 1.19336 \mathrm{e} \quad 638.35441$ 


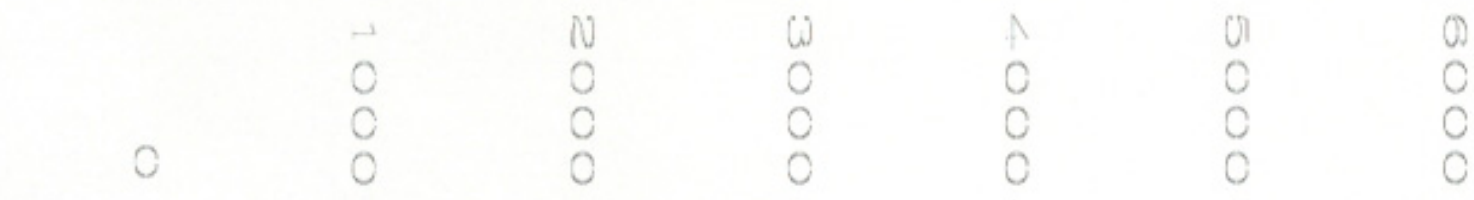

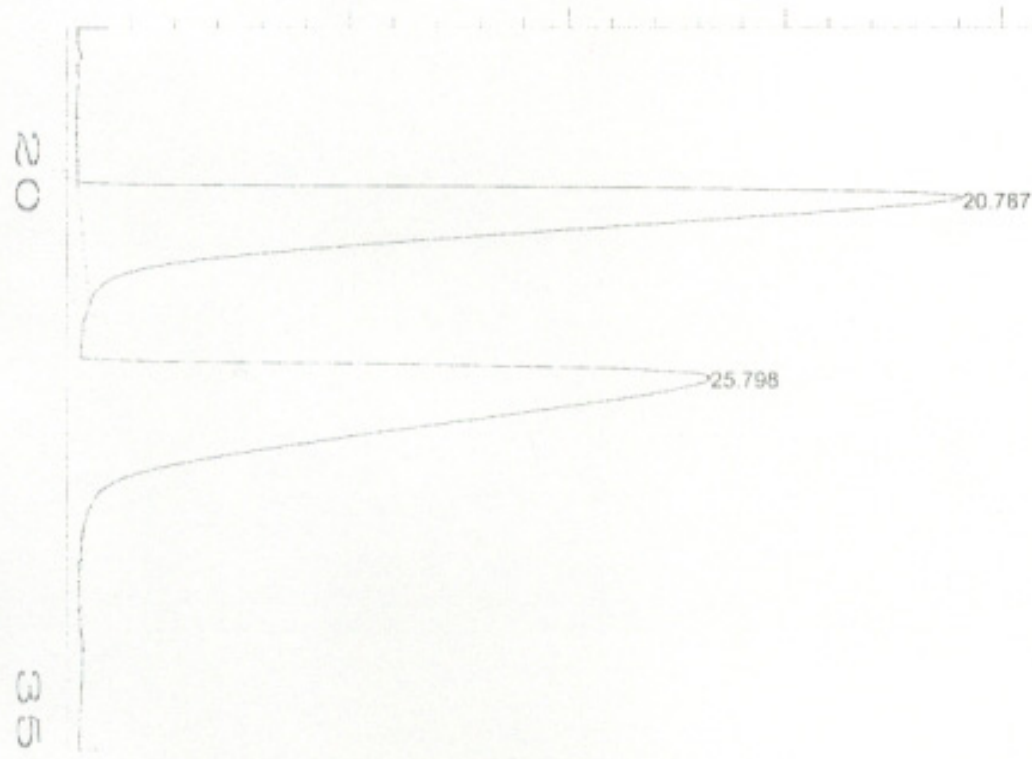

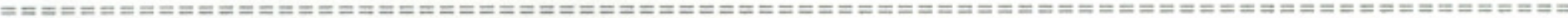
Area Percent Report

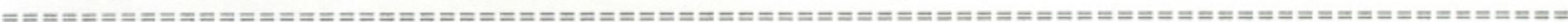

Sig. 1 in $C: \backslash$ PEAK $\backslash$ EXPORT2 $\backslash S I G N A L . D$

\begin{tabular}{ccccccc} 
Pk\# & Ret Time & Area & Height & Type & Width & Area \\
\hline 1 & 20.787 & 322072 & 4058 & BF & 0.945 & 50.2808 \\
2 & 25.798 & 318474 & 2862 & MM & 1.854 & 49.7192
\end{tabular}

Total area $=640546$ 


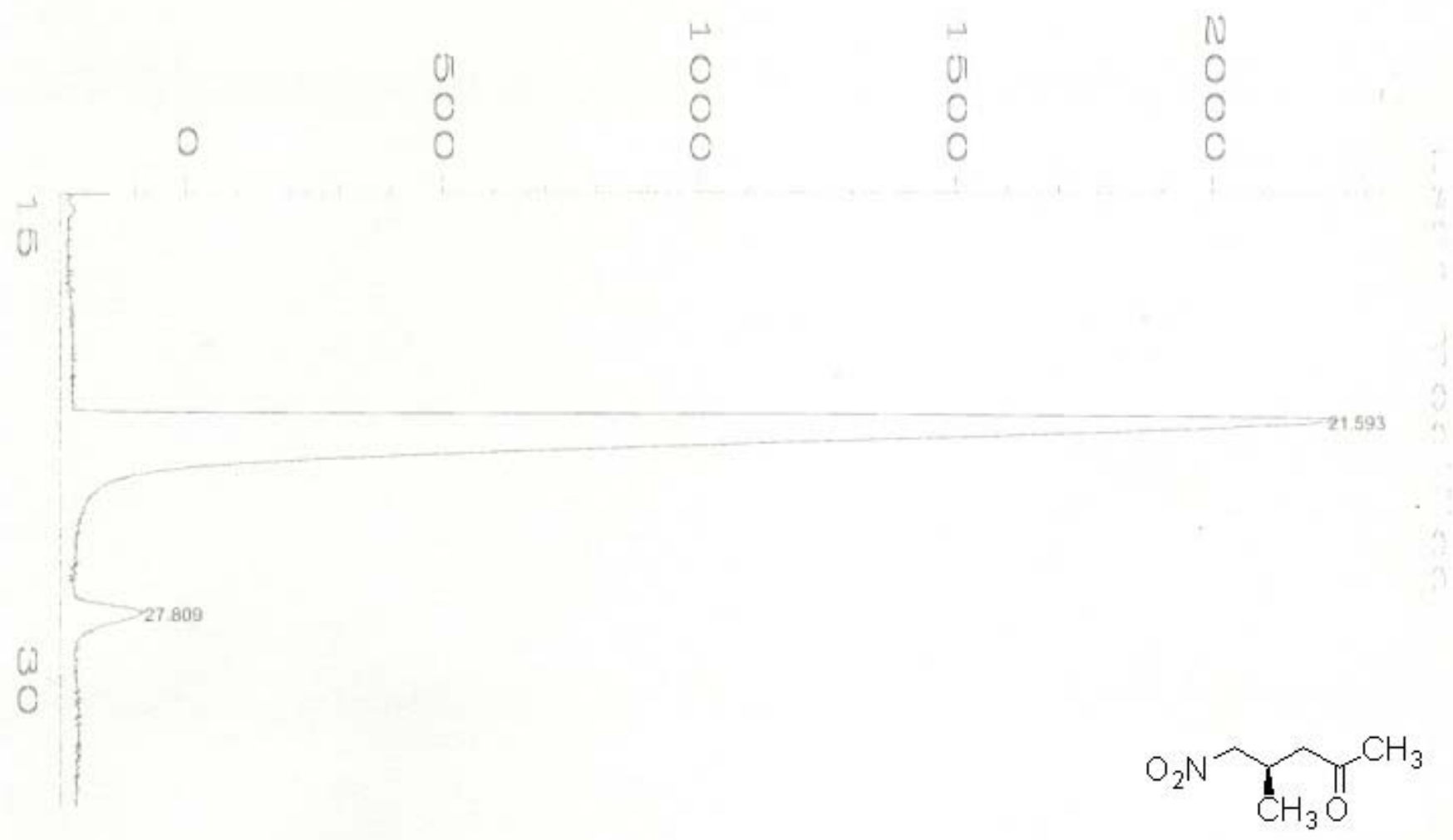

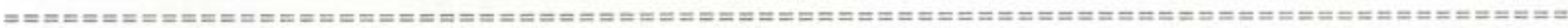
Area Percent Report

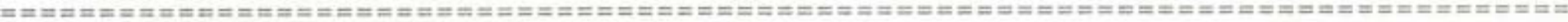

Sig. 1 in C: \PEAK\EXPORT2 \SIGNAL.D

\begin{tabular}{|ccccccc} 
Pk\# & Ret Time & Area & Height & Type & Width & Area \\
\hline 1 & 21.593 & 158702 & 2467 & MM & 1.072 & 96.6305 \\
2 & 27.809 & 5534 & 137 & MM & 0.673 & 3.3695
\end{tabular}

Total area $=164236$ 


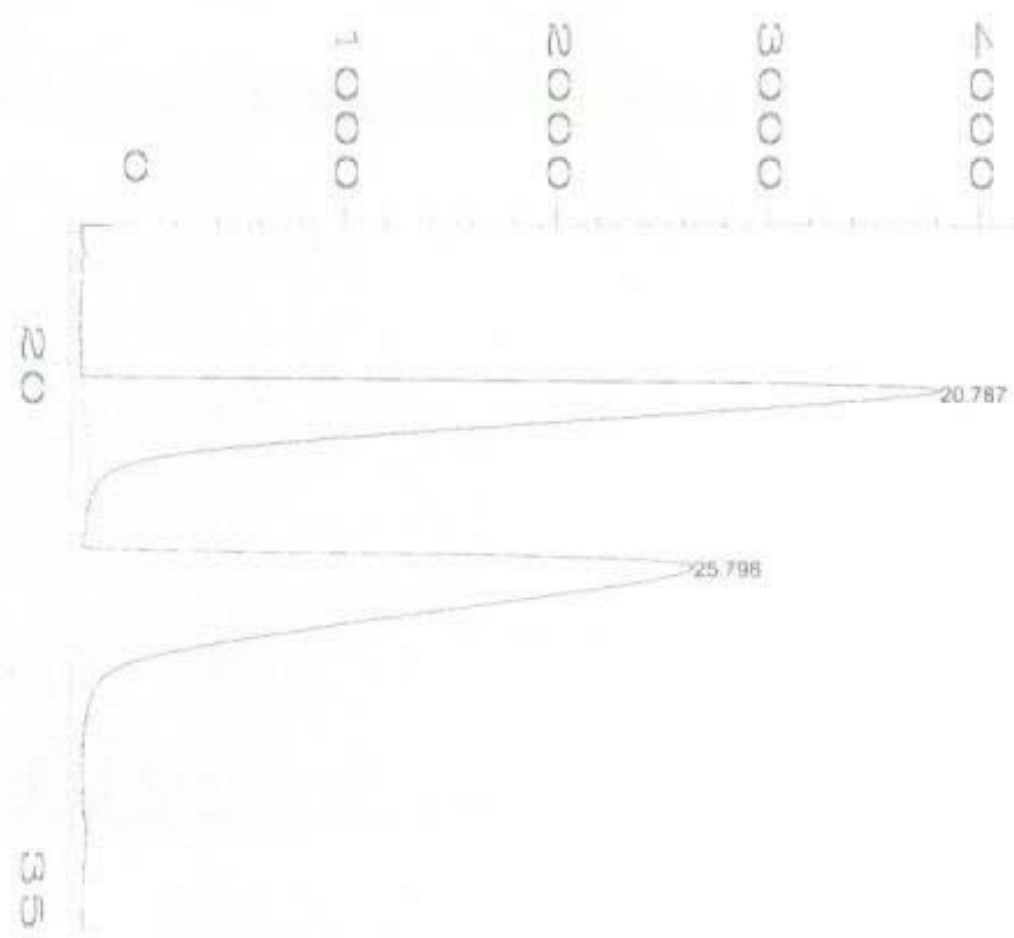

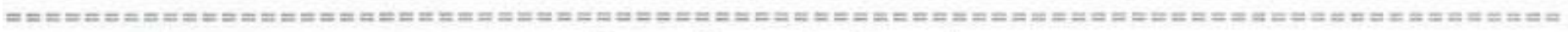

Area Percent Report

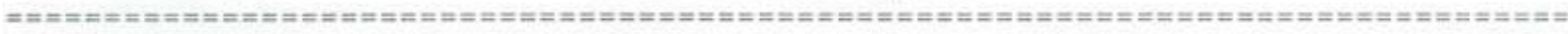

Data File Name : C: \PEAK\EXPORT2 $\backslash S I G N A L . D$

Instrument

Sample Name

HP5890A

Run Time Bar Code:

Acquired on : JAN 15, 1905 10:43:55

Report Created on: 15 Jan 05 11:45 AM

Page Number : 1

Vial Number : 0

Injection Number :

Sequence Line :

Instrument Method:

Analysis Method : QC15MIN.MTH

Sig. 1 in $\mathrm{C}: \backslash$ PEAK \EXPORT2 $\backslash$ SIGNAL.D

\begin{tabular}{ccccccc} 
Pk\# & Ret Time & Area & Height & Type & Width & Area \\
\hline 1 & 20.787 & 322072 & 4058 & BF & 0.945 & 50.2808 \\
2 & 25.798 & 318474 & 2862 & MM & 1.854 & 49.7192
\end{tabular}

Total area $=640546$

User Modified 


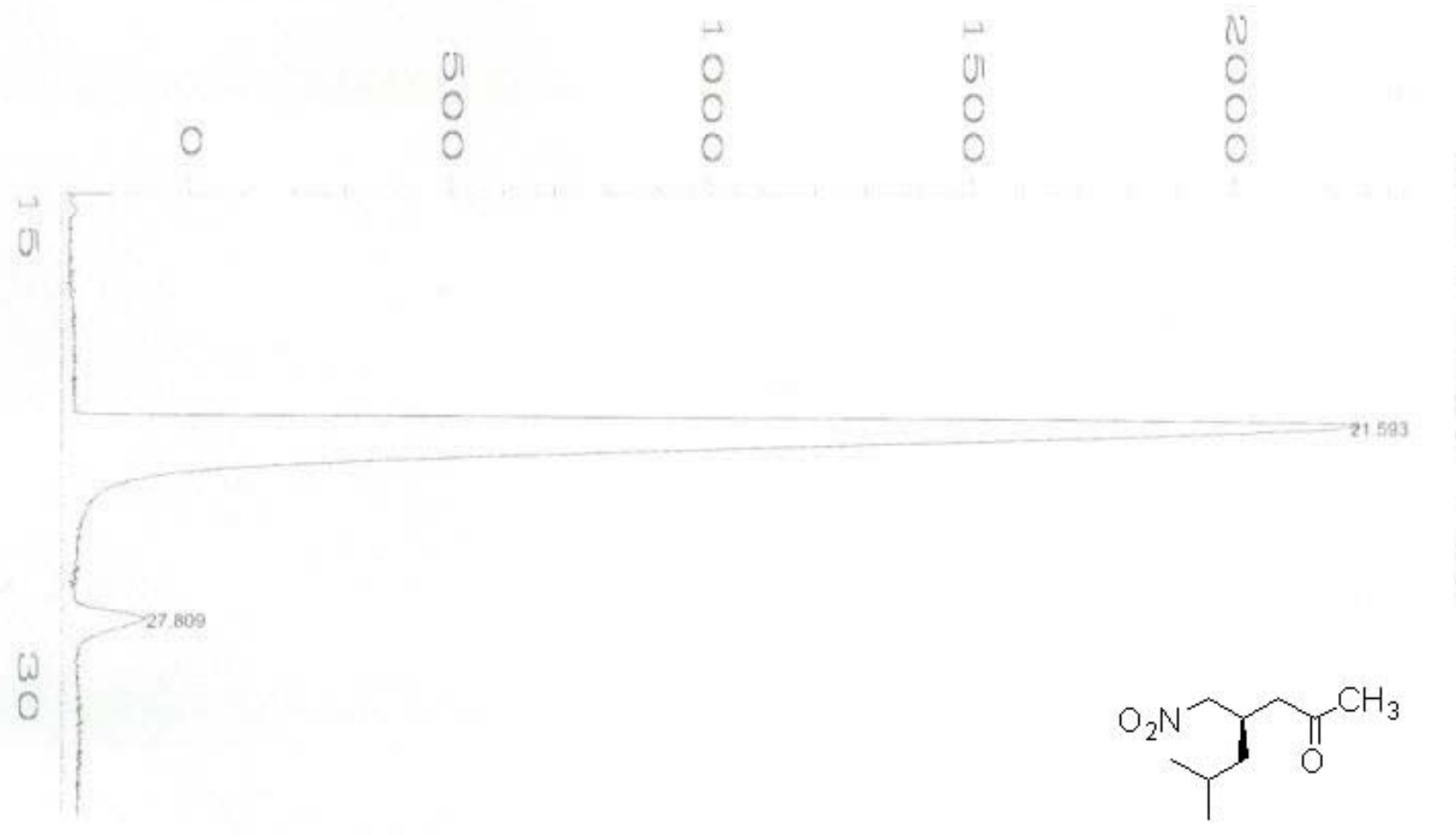

Area Percent Report

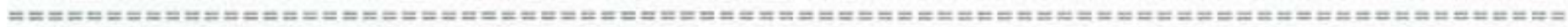

Data File Name :

operator

Instrument

C: \PEAK\EXPORT2\SIGNAL.D

Sample Name :

HP5890A

Run Time Bar Code:

Acquired on : JAN 15, 1905 11:49:33

Report Created on: 15 Jan 05 12:29 PM

Page Number : 1

Vial Number : 0

Injection Number :

Sequence Line :

Instrument Method:

Analysis Method : QC15MIN.MTH

Sig. 1 in $C: \backslash P E A K \backslash E X P O R T 2 \backslash S I G N A L$. D

\begin{tabular}{|ccccccc|} 
Pk\# & Ret Time & Area & Height & Type & Width & Area \\
\hline 1 & 21.593 & 158702 & 2467 & MM & 1.072 & 96.6305 \\
2 & 27.809 & 5534 & 137 & MM & 0.673 & 3.3695
\end{tabular}

Total area $=164236$

User Modified 
gamma TA, 110 isothermic, front column
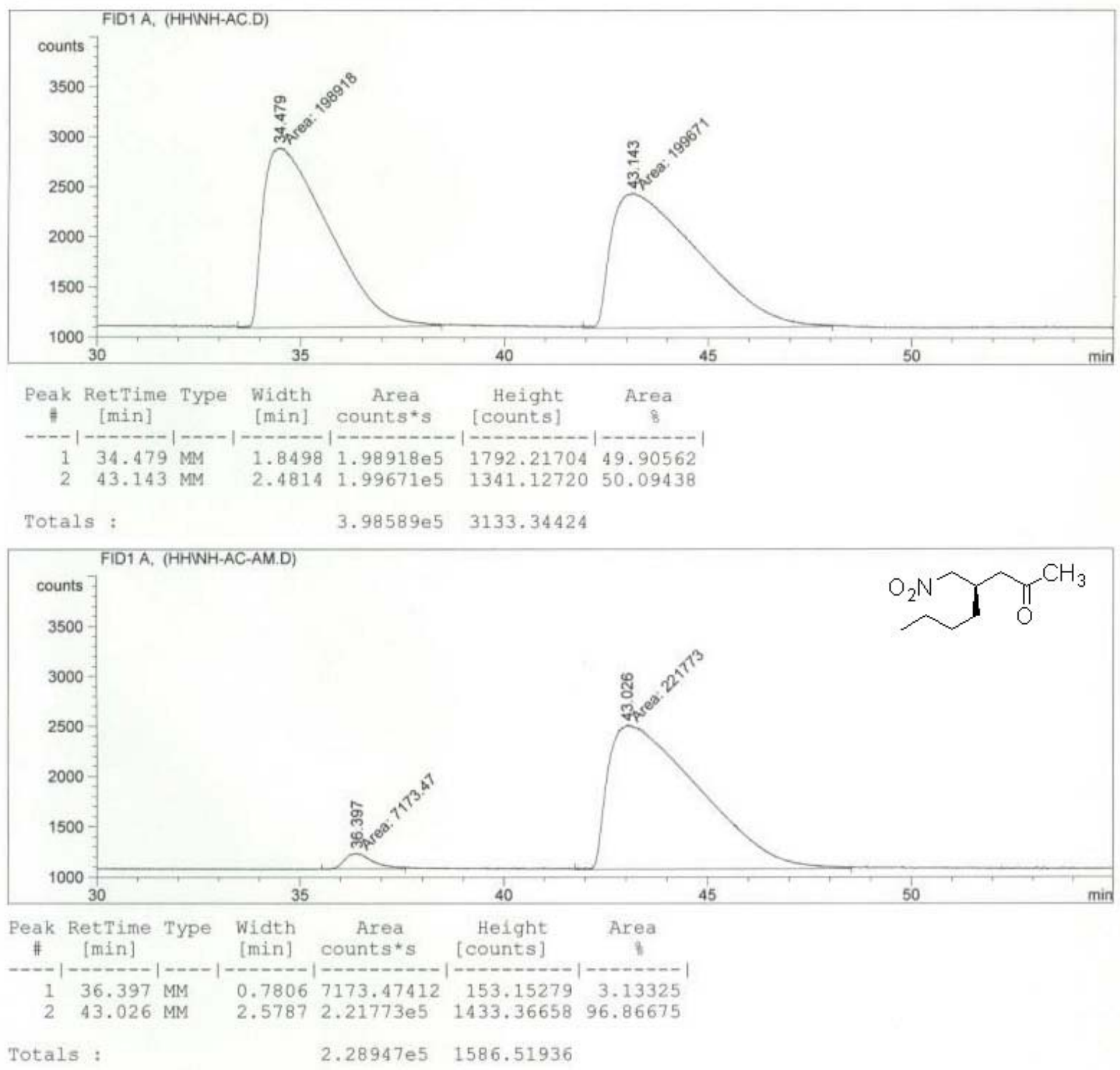


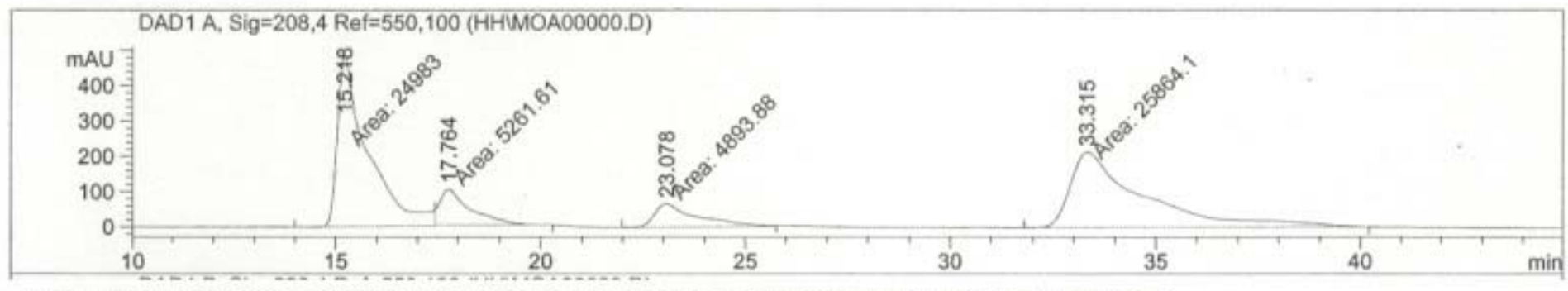

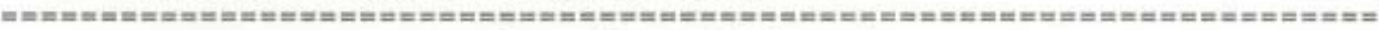

Area Percent Report

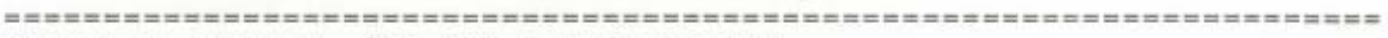

Signal 1: DAD1 A, Sig $=208,4$ Ref $=550,100$

\begin{tabular}{|c|c|c|c|c|c|c|}
\hline $\begin{array}{c}\text { Peak } \\
\#\end{array}$ & $\begin{array}{c}\text { RetTime } \\
\text { [min] }\end{array}$ & Type & $\begin{array}{l}\text { Width } \\
\text { [min] }\end{array}$ & $\begin{array}{c}\text { Area } \\
{\left[m A U^{\star} s\right]}\end{array}$ & $\begin{array}{l}\text { Height } \\
\text { [mAU] }\end{array}$ & $\underset{\frac{q}{8}}{\text { Area }}$ \\
\hline & & & 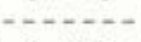 & $\cdots$ & -- & 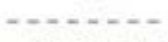 \\
\hline 1 & 15.218 & $\mathrm{MM}$ & 0.8594 & $2.49830 \mathrm{e} 4$ & 484.51413 & 40.9540 \\
\hline 2 & 17.764 & MM & 0.8697 & 5261.61133 & 100.82719 & 8.6252 \\
\hline 3 & 23.078 & $\mathrm{MM}$ & 1.2216 & 4893.88330 & 66.77068 & 8.0224 \\
\hline 4 & 33.315 & MM & 2.0332 & $2.58641 \mathrm{e} 4$ & 212.01376 & 42.3983 \\
\hline
\end{tabular}

Totals :

$6.10026 \mathrm{e} 4 \quad 864.12577$
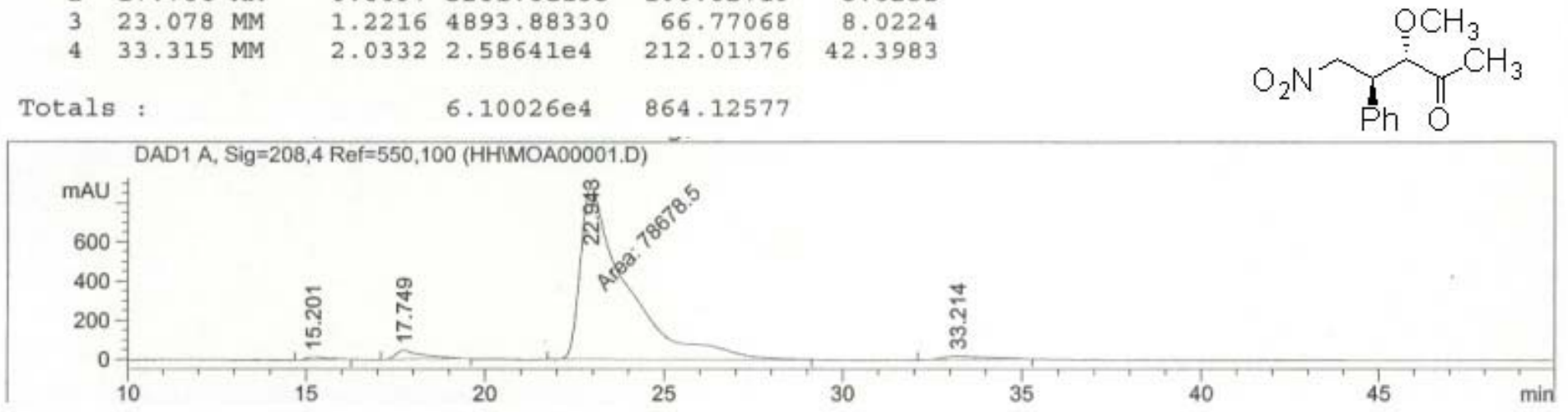

Area Percent Report

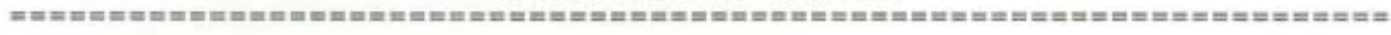

Signal 1: DAD1 A, Sig=208,4 Ref=550,100

\begin{tabular}{|c|c|c|c|c|c|c|}
\hline $\begin{array}{c}\text { Peak } \\
\#\end{array}$ & $\begin{array}{l}\text { RetTime } \\
\text { [min] }\end{array}$ & Type & $\begin{array}{l}\text { Width } \\
\text { [min] }\end{array}$ & $\begin{array}{c}\text { Area } \\
{\left[\mathrm{mAU}^{*} \mathrm{~s}\right]}\end{array}$ & $\begin{array}{l}\text { Height } \\
\text { [mAU] }\end{array}$ & $\begin{array}{c}\text { Area } \\
\frac{o}{6}\end{array}$ \\
\hline & & & & & & \\
\hline 1 & 15. & PB & 882 & 528.36 & 1 & 337 \\
\hline 2 & 9 & VB & 963 & 2526. & 45 . & 03 \\
\hline 3 & 22 & MM & 1. & 7.8678 & 879. & 94. \\
\hline 4 & 33.214 & 2 & 1.0189 & 1640.60608 & 19.23919 & 1.9678 \\
\hline
\end{tabular}

Totals :

$8.33740 \mathrm{e} 4 \quad 956.79876$ 


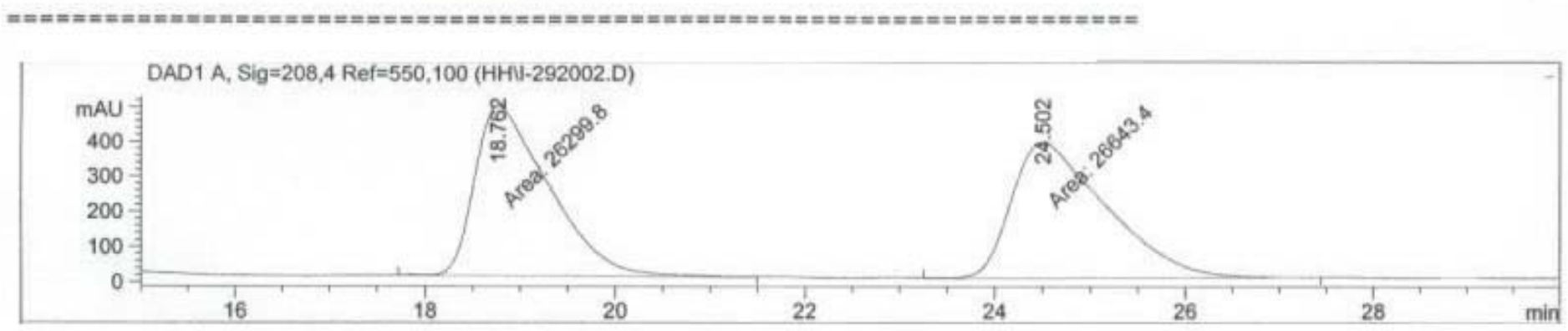

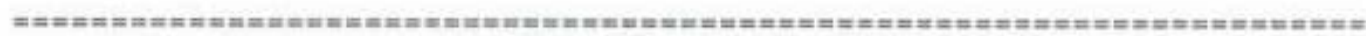

Area Percent Report

\begin{tabular}{|c|c|c|c|c|c|c|}
\hline Peak & $\begin{array}{l}\text { RetTime } \\
\text { [min] }\end{array}$ & Type & $\begin{array}{l}\text { Width } \\
\text { [min] }\end{array}$ & $\begin{array}{c}\text { Area } \\
\text { [mAU*s] }\end{array}$ & $\begin{array}{l}\text { Height } \\
\text { [mAU] }\end{array}$ & $\begin{array}{c}\text { Area } \\
t\end{array}$ \\
\hline & & & & & - n- & $\ldots+\cdots$ \\
\hline 1 & 18.762 & MM & 0.9027 & $2.6299 \mathrm{Be} 4$ & 485.58133 & 49.6755 \\
\hline 2 & 24.502 & MM & 1.1492 & $2.66434 \mathrm{e} 4$ & 386.40411 & 50.3245 \\
\hline Tota] & $8 \div$ & & & $5.29433 \mathrm{e} 4$ & 871.98544 & \\
\hline
\end{tabular}
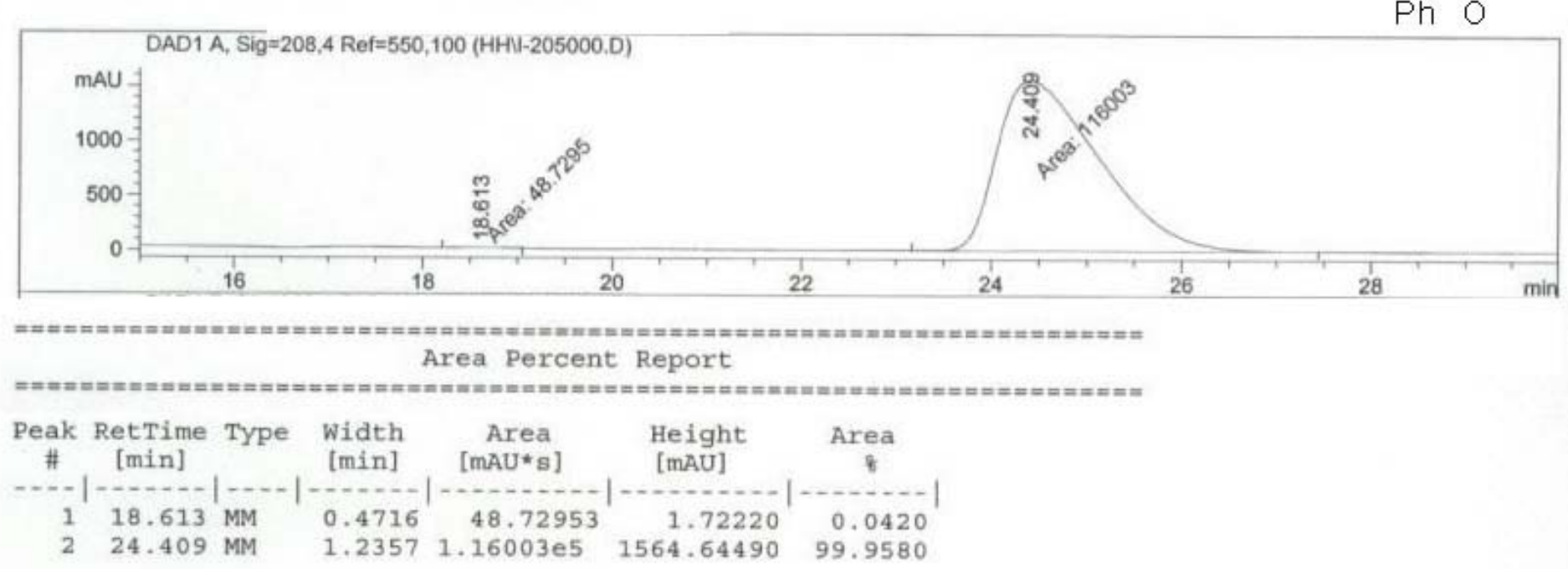

Totals :

$1.16052 \mathrm{e} 51566.36710$ 
FID1 A, (HHUBN-MOA-R.D)
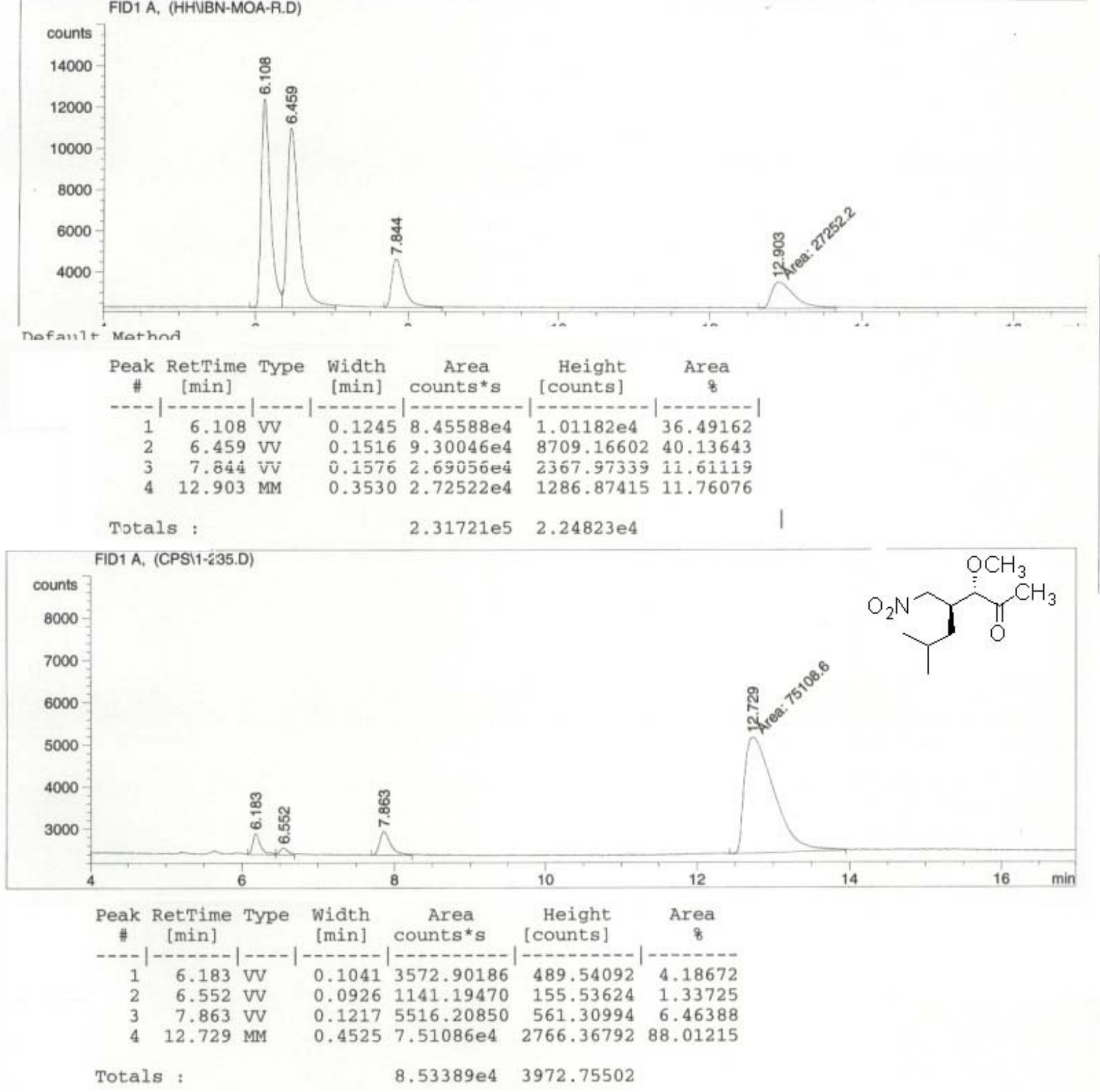

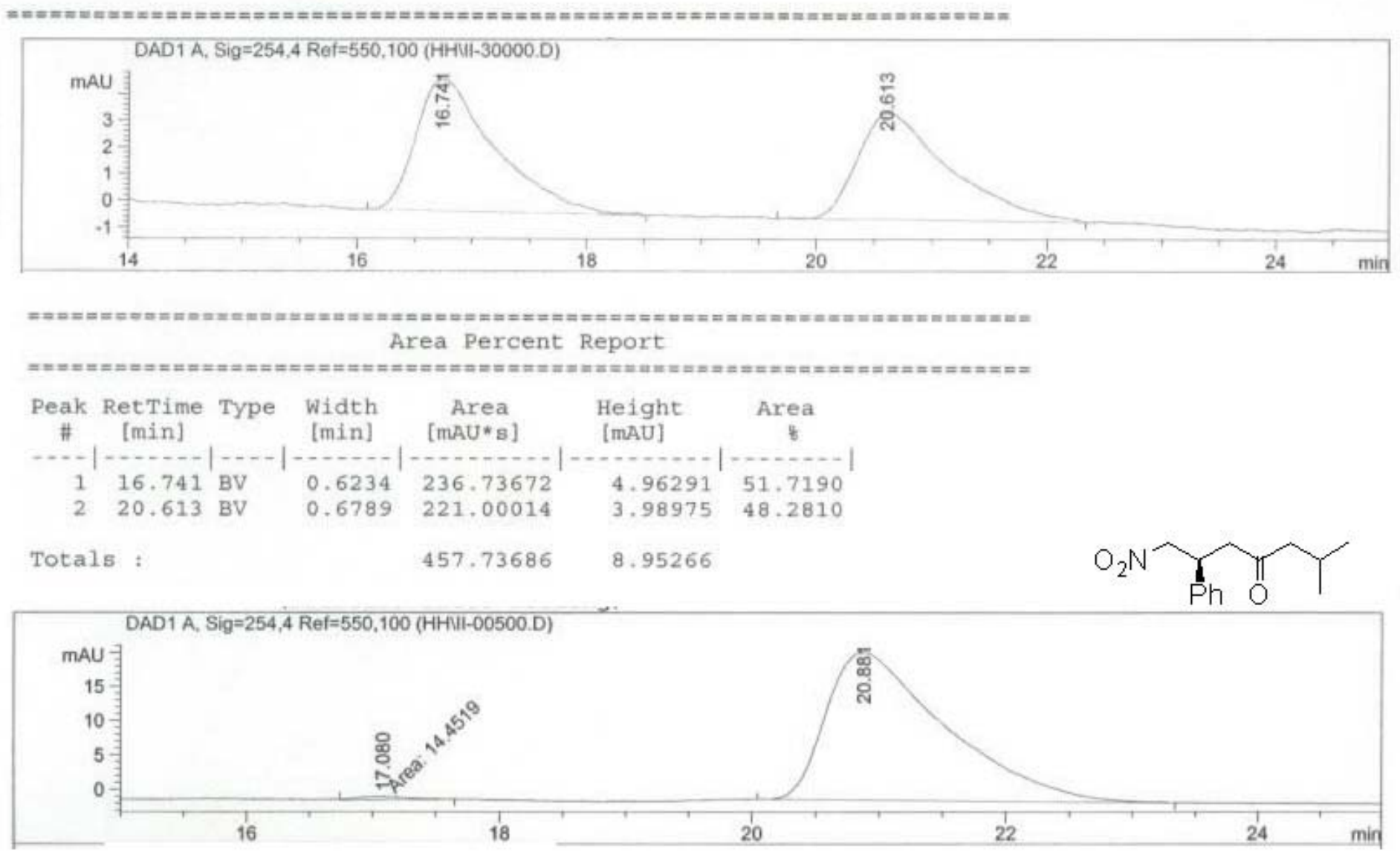

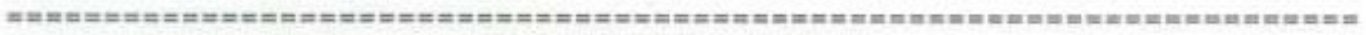

Area Percent Report

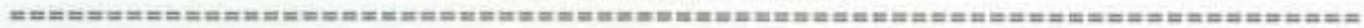

Peak RetTime Type width Area Height Area

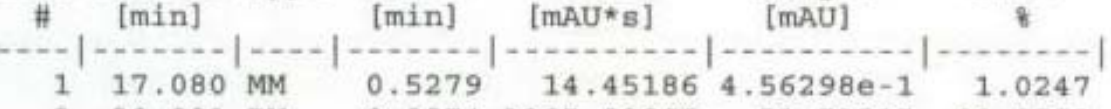

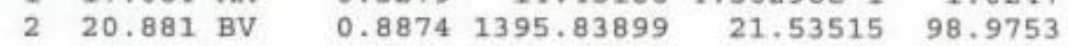

Totals : $\quad 1410.29085 \quad 21.99145$ 
DAD1 D, Sig=208,4 Ref=550,100 (HHUI065300.D)

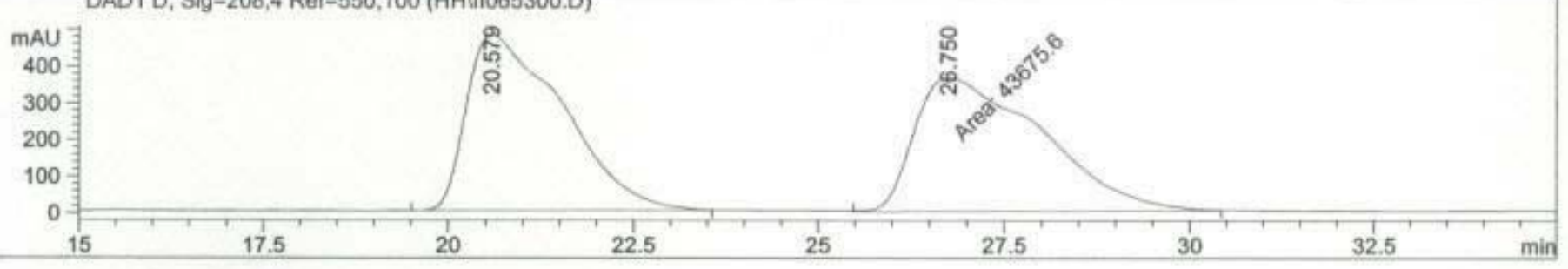

Area Percent Report

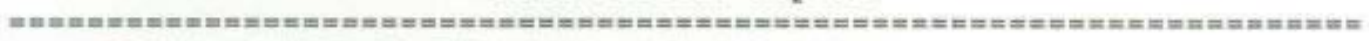

Signal 3: DAD1 D, Sig=208, 4 Ref $=550,100$

Peak RetTime Type width Area Height Area

\# [min] [min] [mAU*s] [mAU] है

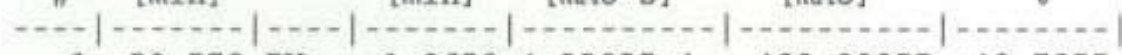

$\begin{array}{lllllll}1 & 20.579 & \text { PV } & 1.0650 & 4.33025 \mathrm{e} 4 & 480.00055 & 49.7855\end{array}$

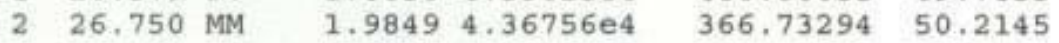

Totals ;

$8.69781 e 4 \quad 846.73349$

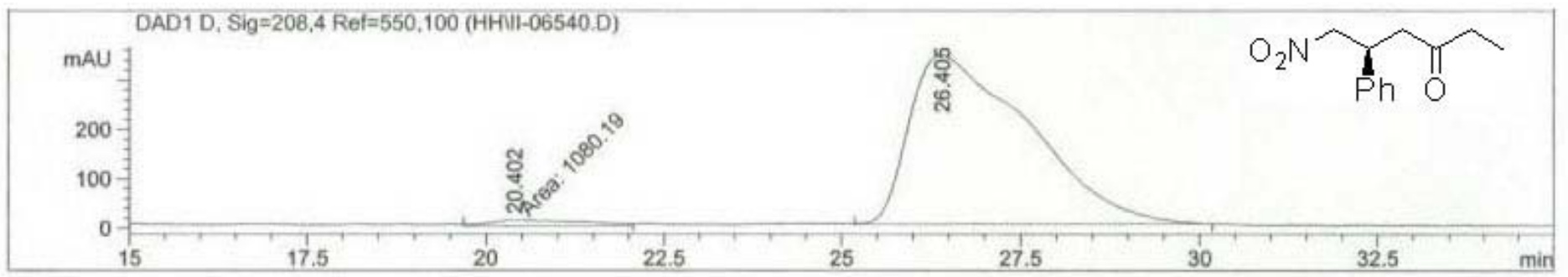

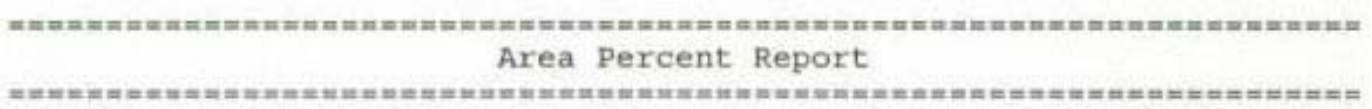

Signal 3: DAD1 D, Sig=208, 4 Ref $=550,100$

Peak RetTime Type Width Area Height Area

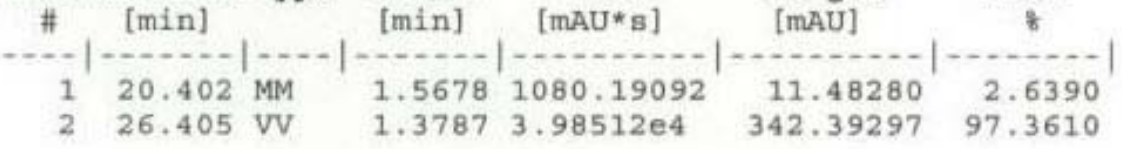

Totals : $\quad 4.09314 \mathrm{e} 4 \quad 353.87577$ 
10* ipa/hex, $1 \mathrm{~mL} / \mathrm{min}$, OD
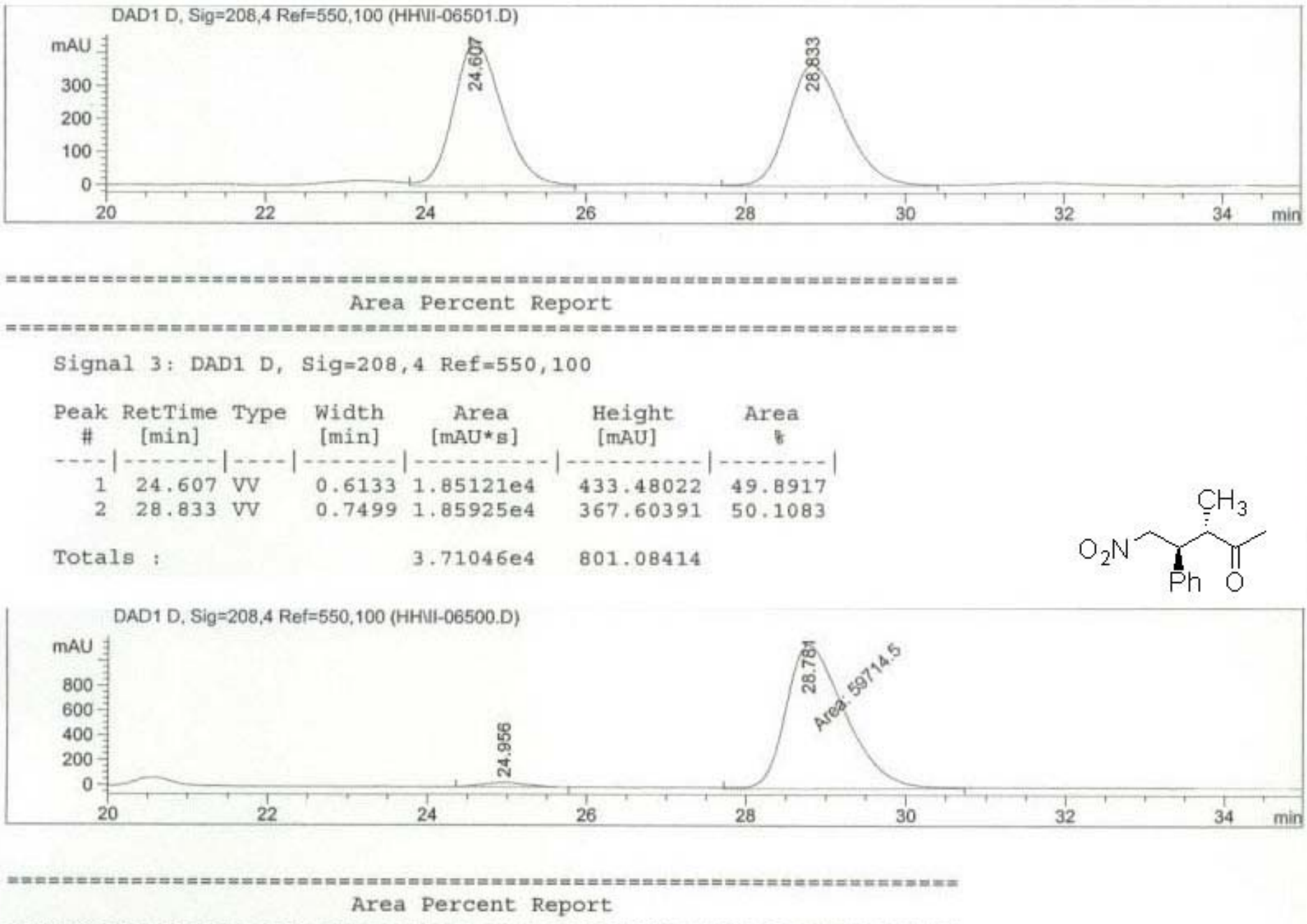

Signal 3: DAD1 D, $S i g=208,4$ Ref $=550,100$

\begin{tabular}{|c|c|c|c|c|c|c|}
\hline $\begin{array}{c}\text { Peak } \\
\#\end{array}$ & $\begin{array}{l}\text { RetTime } \\
\text { [min] }\end{array}$ & Type & $\begin{array}{l}\text { Width } \\
\text { [min] }\end{array}$ & $\begin{array}{c}\text { Area } \\
{\left[m A U^{*} s\right]}\end{array}$ & $\begin{array}{l}\text { Height } \\
\text { [mAU] }\end{array}$ & $\underset{\text { fo }}{\text { Area }}$ \\
\hline & & & & & & \\
\hline $\begin{array}{l}1 \\
2\end{array}$ & $\begin{array}{l}24.956 \\
28.781\end{array}$ & MV & $\begin{array}{l}0.4924 \\
0.8589\end{array}$ & $\begin{array}{l}1418.16736 \\
5.97145 \mathrm{e}\end{array}$ & $\begin{array}{r}35.52486 \\
1158.70398\end{array}$ & $\begin{array}{r}2.3198 \\
97.6802\end{array}$ \\
\hline otal & 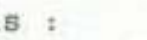 & & & $6.11327 \mathrm{e} 4$ & 1194.22884 & \\
\hline
\end{tabular}


$O D, 108 \mathrm{ipa} / \mathrm{hex}, 1.0 \mathrm{~mL} / \mathrm{min}$

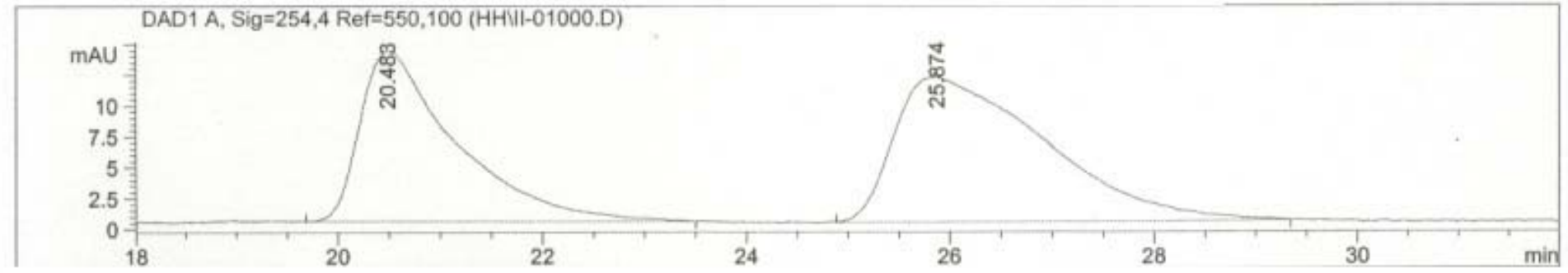

Area Percent Report

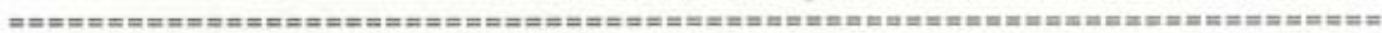

Signal 3: DAD1 D, Sig=208, 4 Ref $=550,100$

\begin{tabular}{|c|c|c|c|c|c|c|}
\hline $\begin{array}{c}\text { Peak } \\
\#\end{array}$ & $\begin{array}{l}\text { RetTime } \\
\text { [min] }\end{array}$ & Type & $\begin{array}{l}\text { Width } \\
\text { [min] }\end{array}$ & $\begin{array}{c}\text { Area } \\
{\left[\mathrm{mAU}{ }^{*} \mathrm{~s}\right]}\end{array}$ & $\begin{array}{l}\text { Height } \\
\text { [mAU] }\end{array}$ & $\begin{array}{c}\text { Area } \\
\text { के }\end{array}$ \\
\hline & & & & & & \\
\hline $\begin{array}{l}1 \\
2\end{array}$ & $\begin{array}{l}20.489 \\
25.770\end{array}$ & $\begin{array}{l}\text { MM } \\
\text { VV }\end{array}$ & $\begin{array}{l}1.0833 \\
1.0288\end{array}$ & $\begin{array}{l}4.46746 \mathrm{e} 4 \\
4.30558 \mathrm{e} 4\end{array}$ & $\begin{array}{l}687.29510 \\
545.30322\end{array}$ & $\begin{array}{l}50.9226 \\
49.0774\end{array}$ \\
\hline
\end{tabular}

Totals :

$8.77304 \mathrm{e} \quad 1232.59833$
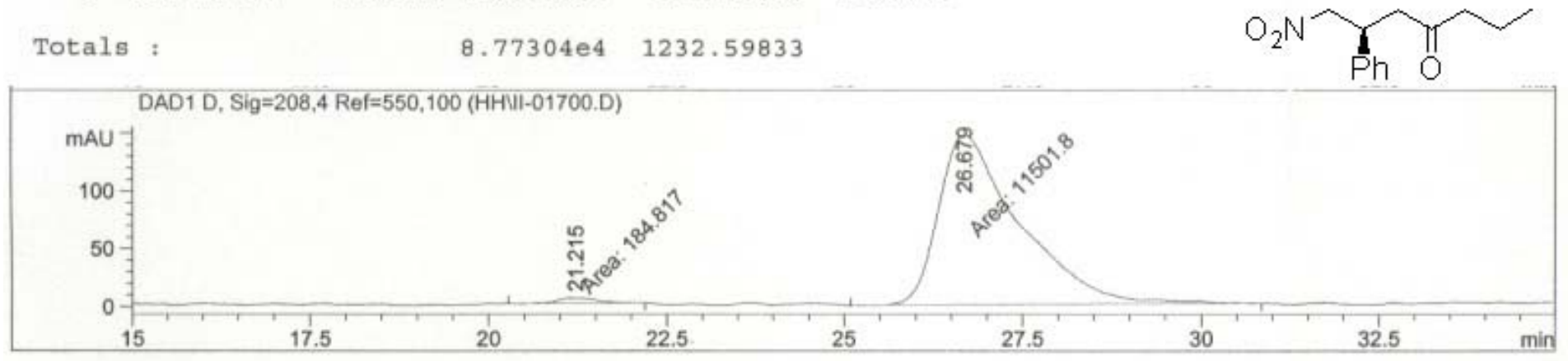

Area Percent Report

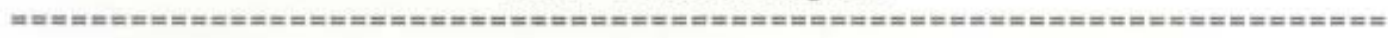

Signal 3: DAD1 D, Sig=208, 4 Ref $=550,100$

\begin{tabular}{|c|c|c|c|c|c|c|}
\hline $\begin{array}{c}\text { Peak } \\
\#\end{array}$ & $\begin{array}{l}\text { RetTime } \\
\text { [min] }\end{array}$ & Type & $\begin{array}{l}\text { Width } \\
\text { [min] }\end{array}$ & $\begin{array}{c}\text { Area } \\
{\left[\mathrm{mAU}^{\star} \mathrm{s}\right]}\end{array}$ & $\begin{array}{l}\text { Height } \\
\text { [mAU] }\end{array}$ & $\underset{\text { Area }}{\text { of }}$ \\
\hline 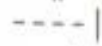 & & & & & $\ldots$ & (n- \\
\hline 1 & 21.215 & $\mathrm{MM}$ & 0.6185 & 184.81703 & 4.98020 & 1.5814 \\
\hline 2 & 26.679 & MM & 1.3092 & $1.15018 \mathrm{e} 4$ & 146.42561 & 98.4186 \\
\hline
\end{tabular}

Totals :

$1.16866 \mathrm{e} 4$

151.40582 
OD, 10 \& $\mathrm{ipa} / \mathrm{hex}, 1.0 \mathrm{~mL} / \mathrm{min}$

DAD1 D, Sig=208,4 Ref $=550,100$ (HHNII-01100.D)
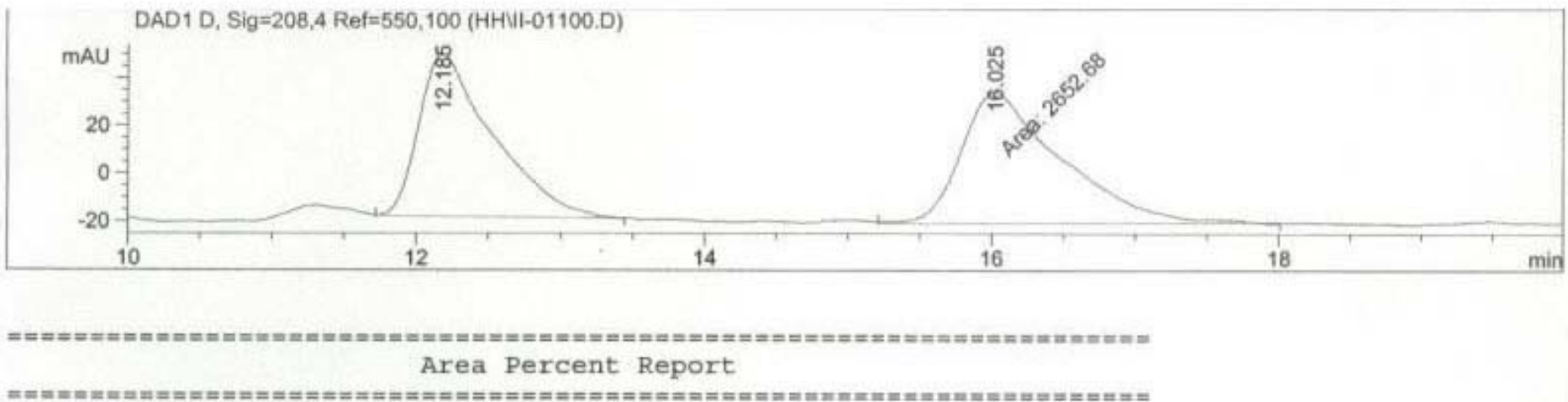

Signal 3: DAD1 D, $S$ ig $=208,4$ Ref $=550,100$

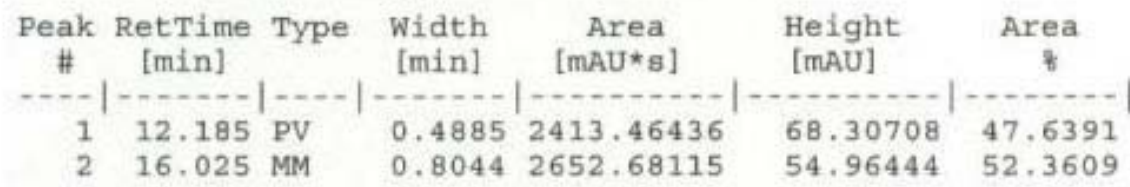

Totals :

$5066.14551 \quad 123.27152$

DAD1 C, Sig=220,4 Ref=550,100 (G:HHHIHPLCIHHUI-01100.D)

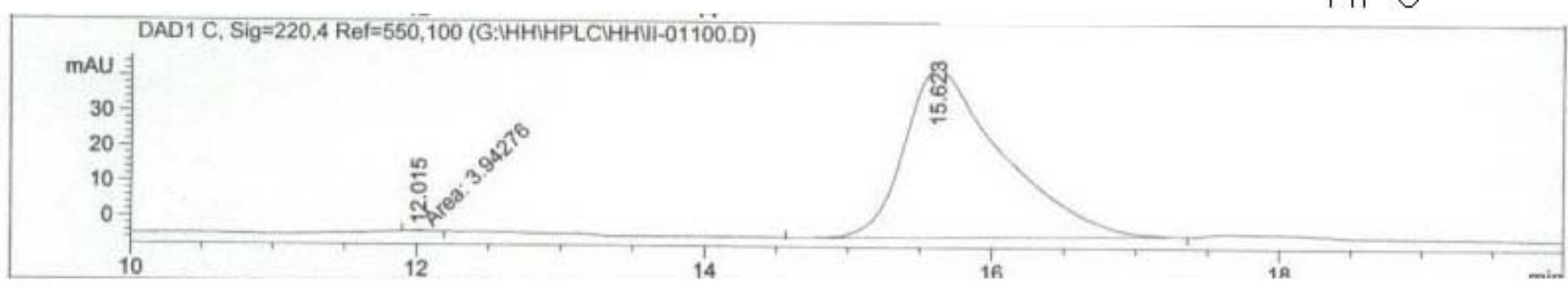

\begin{tabular}{|c|c|c|c|c|c|}
\hline $\begin{array}{c}\text { Peak } \\
\#\end{array}$ & $\begin{array}{l}\text { RetTime Type } \\
\text { [min] }\end{array}$ & $\begin{array}{l}\text { Width } \\
\text { [min] }\end{array}$ & $\begin{array}{c}\text { Area } \\
{\left[m A U^{*} B\right]}\end{array}$ & $\begin{array}{l}\text { Height } \\
\text { [mAU] }\end{array}$ & $\begin{array}{c}\text { Area } \\
\text { कै }\end{array}$ \\
\hline & & & & & \\
\hline $\begin{array}{l}1 \\
2\end{array}$ & $\begin{array}{l}12.015 \mathrm{MM} \\
15.623 \mathrm{pV}\end{array}$ & $\begin{array}{l}0.1981 \\
0.6516\end{array}$ & $\begin{array}{r}3.94276 \\
2294.07373\end{array}$ & 3. $\begin{array}{r}31634 \mathrm{e}-1 \\
48.26081\end{array}$ & $\begin{array}{r}0.1716 \\
99.8284\end{array}$ \\
\hline
\end{tabular}


DAD1 D, Sig=208,4 Ref $=550,100$ (HHUI-056B0.D)

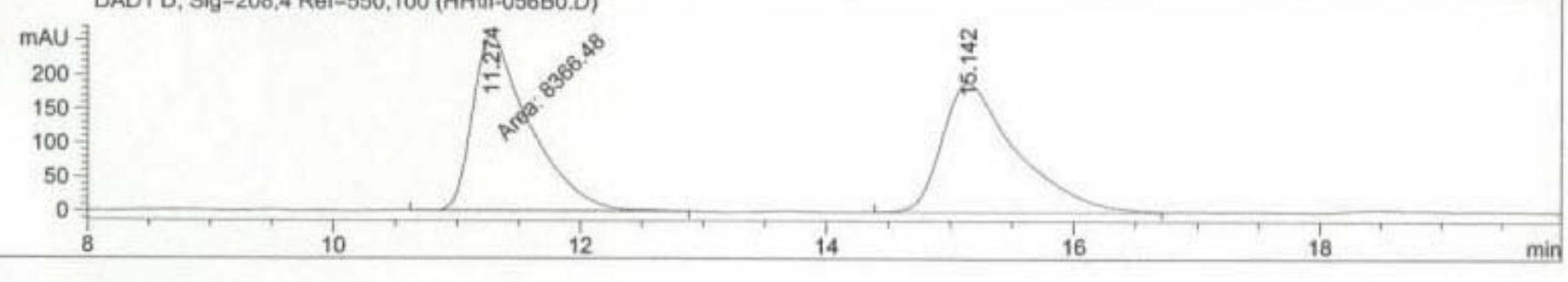

$$
\text { Area Percent Report }
$$

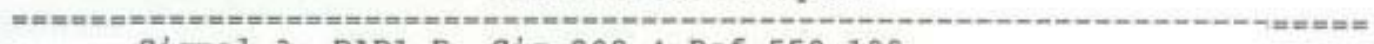

Signal 3: DAD1 D, Sig $=208,4$ Ref $=550,100$

\begin{tabular}{|c|c|c|c|c|c|c|}
\hline $\begin{array}{c}\text { Peak } \\
\#\end{array}$ & $\begin{array}{l}\text { RetTime } \\
\text { [min] }\end{array}$ & Type & $\begin{array}{c}\text { Width } \\
\text { [min] }\end{array}$ & $\begin{array}{c}\text { Area } \\
{[\mathrm{mAU} * \mathrm{~s}]}\end{array}$ & $\begin{array}{l}\text { Height } \\
\text { [mAU] }\end{array}$ & $\begin{array}{c}\text { Area } \\
\frac{8}{6}\end{array}$ \\
\hline 1 & 11.274 & MM & 0.5399 & 8366.47559 & 258.28879 & 50.9729 \\
\hline 2 & 15.142 & VV & 0.5788 & 8047.09766 & 190.41586 & 49.0271 \\
\hline
\end{tabular}

Totals :

$1.64136 \mathrm{e} 4 \quad 448,70465$

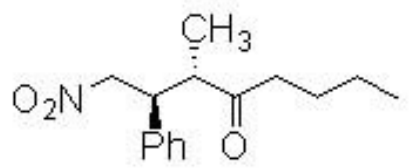

DAD1 D, Sig=208,4 Ref $=550,100$ (HHUII-056A0.D)

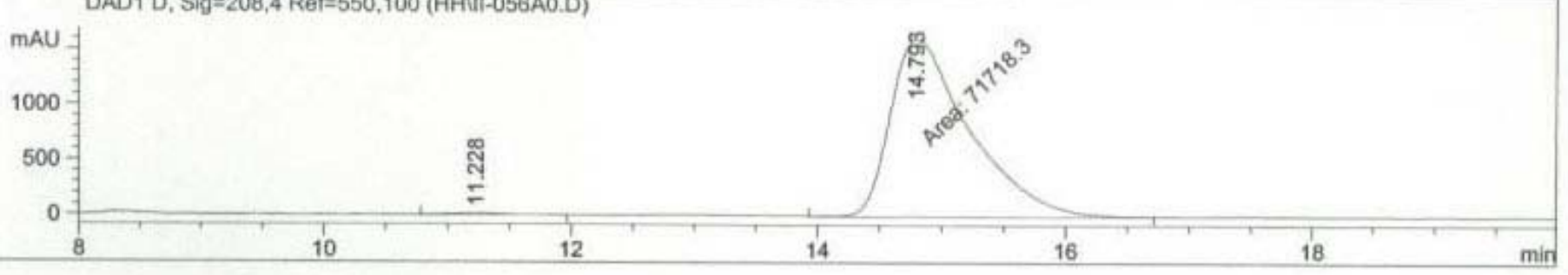

Area Percent Report

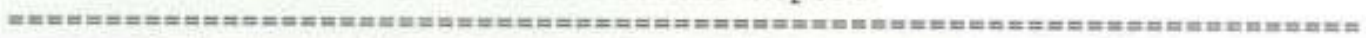

Signal 3: DAD1 D, Sig $=208,4$ Ref $=550,100$

\begin{tabular}{|c|c|c|c|c|c|c|}
\hline $\begin{array}{c}\text { Peak } \\
\#\end{array}$ & $\begin{array}{l}\text { RetTime } \\
\text { [min] }\end{array}$ & Type & $\begin{array}{l}\text { Width } \\
\text { [min] }\end{array}$ & $\begin{array}{c}\text { Area } \\
{\left[m A U^{*} s\right]}\end{array}$ & $\begin{array}{l}\text { Height } \\
\text { [mAU] }\end{array}$ & 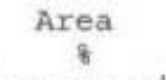 \\
\hline 1 & 11.228 & VV & 0.4008 & 527.28949 & 16.21629 & $0.7299^{\circ}$ \\
\hline 2 & 14.793 & MM & 0.7419 & $7.17183 \mathrm{e} 4$ & 1611.21106 & 99.2701 \\
\hline bta & : & & & $7.22456 \mathrm{e} 4$ & 1627.42735 & \\
\hline
\end{tabular}


g-TA, 120 isothermal, signa12

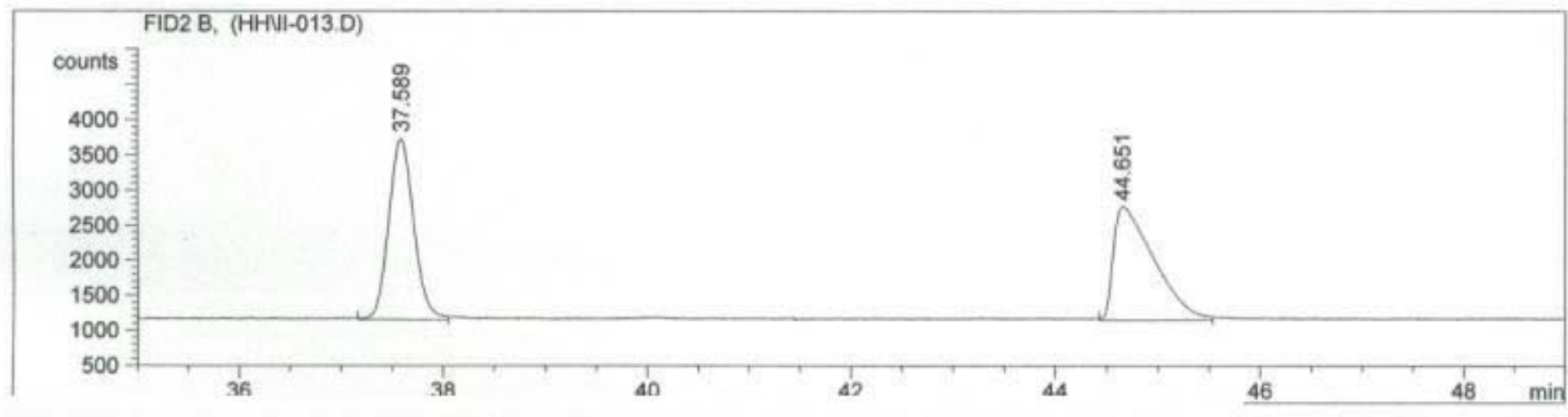

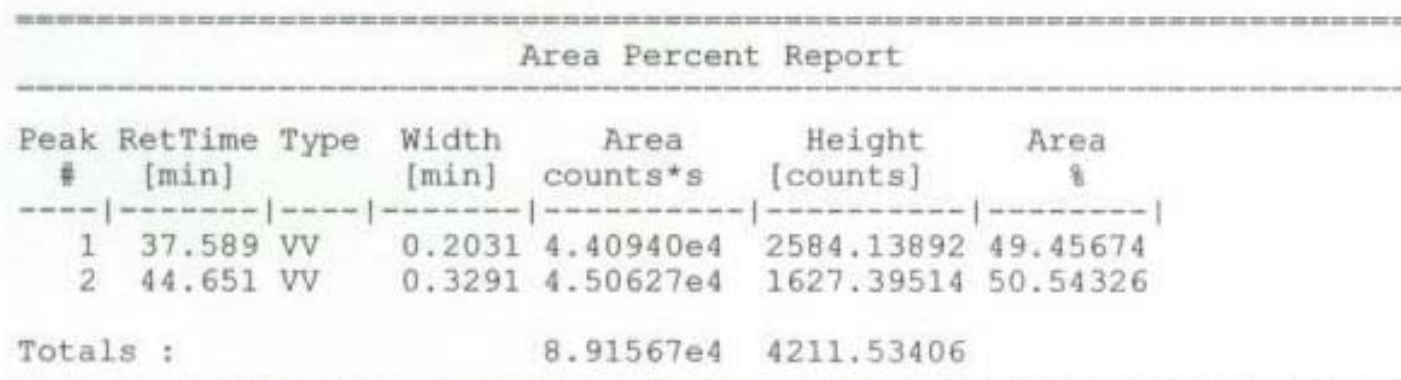

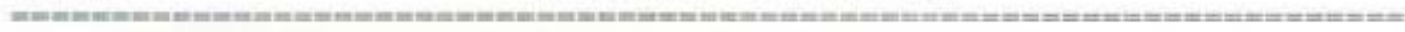

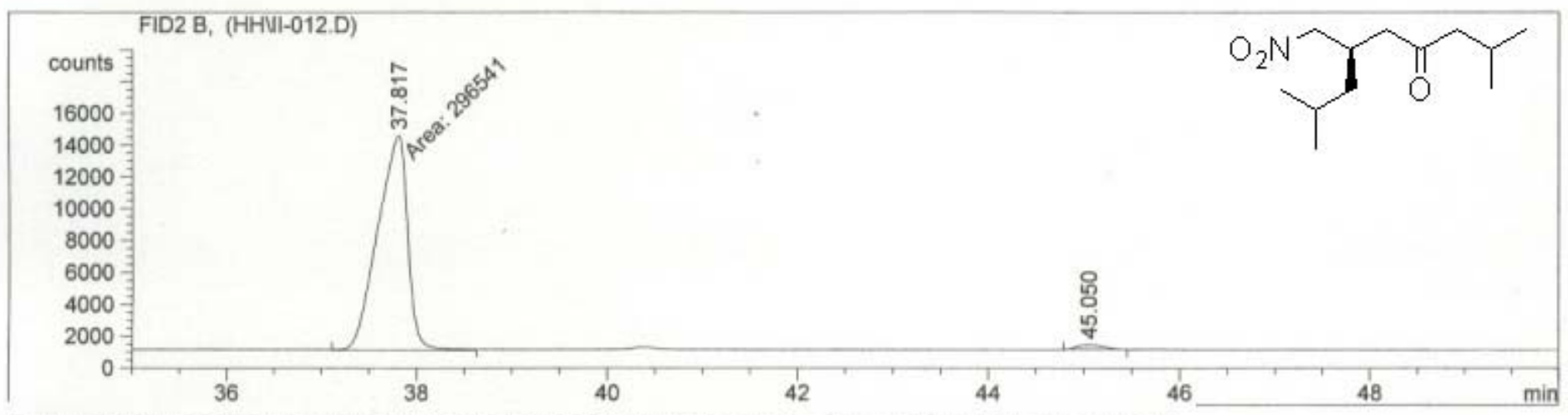

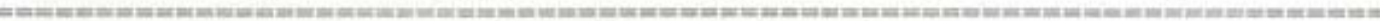

Area Percent Report

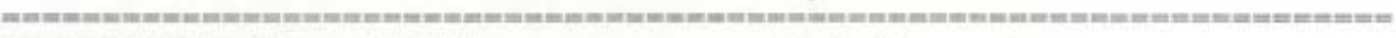

\begin{tabular}{|c|c|c|c|c|c|c|}
\hline $\begin{array}{c}\text { Peak } \\
\#\end{array}$ & $\begin{array}{l}\text { RetTime } \\
\text { [min] }\end{array}$ & Type & $\begin{array}{l}\text { Width } \\
\text { [min] }\end{array}$ & $\begin{array}{c}\text { Area } \\
\text { counts*s }\end{array}$ & $\begin{array}{r}\text { Height } \\
\text { [counts] }\end{array}$ & $\begin{array}{c}\text { Area } \\
\quad 8\end{array}$ \\
\hline $\begin{array}{l}1 \\
2\end{array}$ & $\begin{array}{l}37.817 \\
45.050\end{array}$ & $\begin{array}{l}\text { MM } \\
\text { VV }\end{array}$ & $\begin{array}{l}0.3666 \\
0.2140\end{array}$ & $\begin{array}{l}2.96541 \mathrm{e} 5 \\
4838.98389\end{array}$ & $\begin{array}{c}1.34831 \mathrm{e} 4 \\
269.15033\end{array}$ & $\begin{array}{r}98.39439 \\
1.60561\end{array}$ \\
\hline
\end{tabular}

Totals : $\quad 3.01380 \mathrm{e} 5 \quad 1.37522 \mathrm{e} 4$ 
g-TA, 120 isothermal

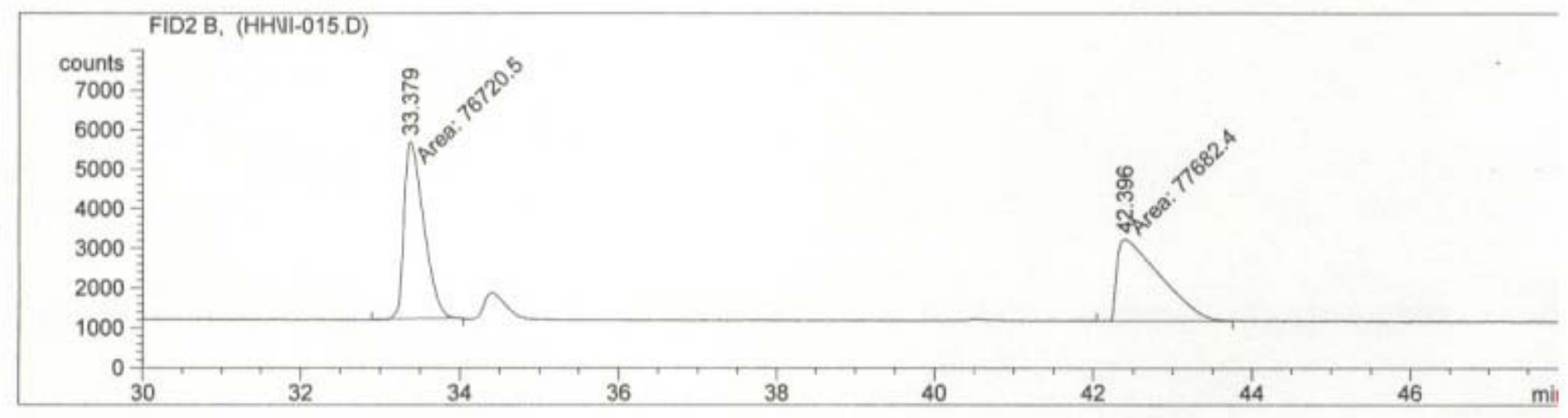

\begin{tabular}{|c|c|c|c|c|c|c|}
\hline $\begin{array}{c}\text { Peak } \\
\#\end{array}$ & $\begin{array}{l}\text { RetTime } \\
\text { [min] }\end{array}$ & Type & $\begin{array}{l}\text { Width } \\
\text { [min] }\end{array}$ & $\begin{array}{c}\text { Area } \\
\text { counts }{ }^{\star} s\end{array}$ & $\begin{array}{r}\text { Height } \\
\text { [counts] }\end{array}$ & $\begin{array}{c}\text { Area } \\
8\end{array}$ \\
\hline $\begin{array}{l}1 \\
2\end{array}$ & $\begin{array}{l}33.379 \\
42.396\end{array}$ & $\begin{array}{l}\text { MM } \\
\text { MM }\end{array}$ & $\begin{array}{l}0.2864 \\
0.6301\end{array}$ & $\begin{array}{l}7.67205 \mathrm{e} 4 \\
7.76824 \mathrm{e} 4\end{array}$ & $\begin{array}{l}4465.04443 \\
2054.86279\end{array}$ & $\begin{array}{l}49.68851 \\
50.31149\end{array}$ \\
\hline Total & : & & & $1.54403 \mathrm{e} 5$ & 6519.90723 & \\
\hline
\end{tabular}

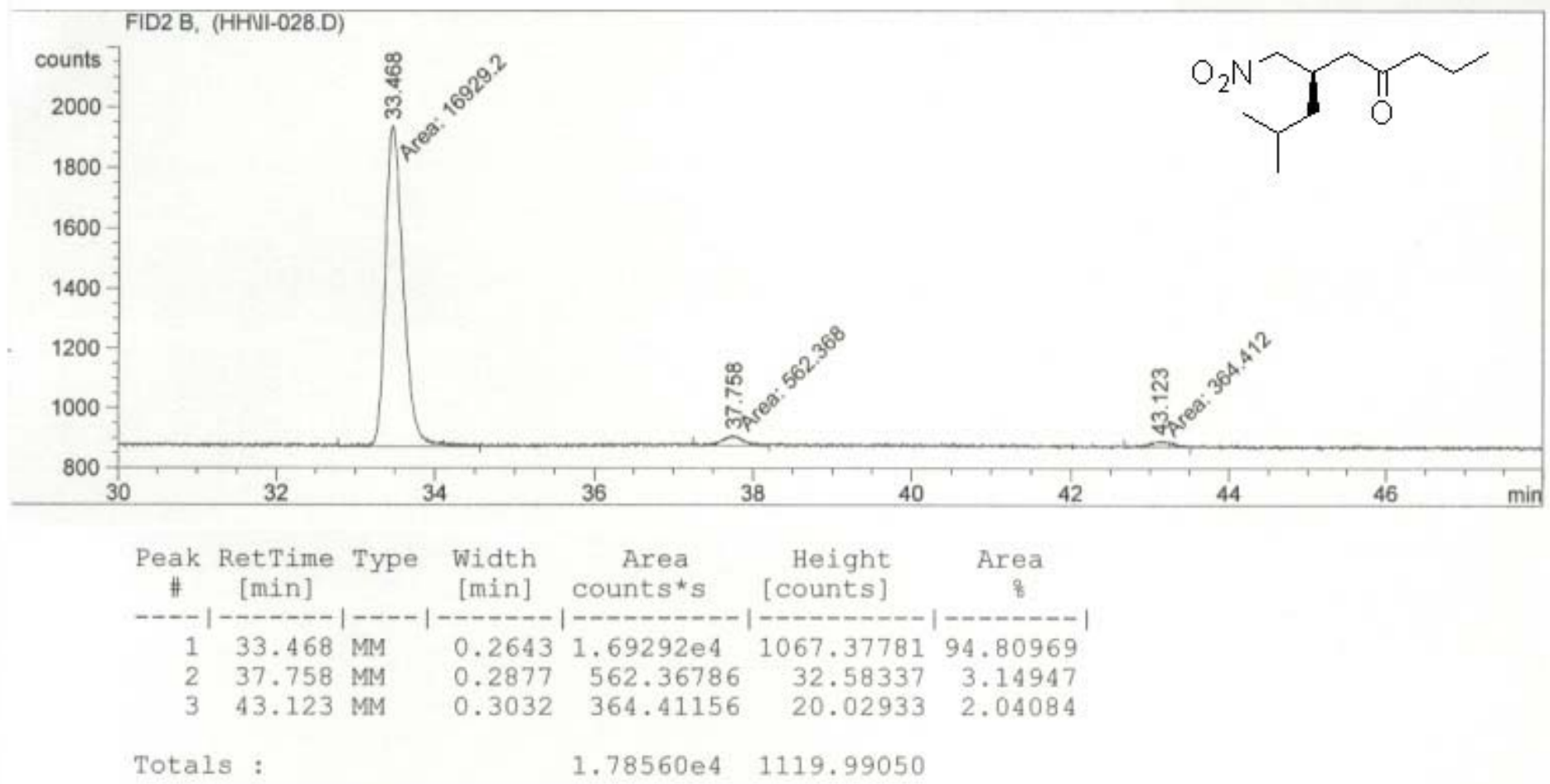

